\title{
Adults adapt to child speech in semantic use
}

\author{
Guanghao You ${ }^{1,2}$, Moritz M. Daum ${ }^{2,3,4}$, and Sabine Stoll ${ }^{1,2}$ \\ ${ }^{1}$ Department of Comparative Language Science, University of Zurich \\ ${ }^{2}$ Center for the Interdisciplinary Study of Language Evolution (ISLE), University of Zurich \\ ${ }^{3}$ Department of Psychology, University of Zurich \\ ${ }^{4}$ Jacobs Center for Productive Youth Development, University of Zurich
}

\begin{abstract}
Author Note
Correspondence concerning this article should be addressed to Guanghao You, Department of Comparative Language Science, University of Zurich, Thurgauerstrasse 30, 8050 Zurich, Switzerland. E-mail: guanghao.you@uzh.ch

Moritz M. Daum and Sabine Stoll share the last authorship.

The authors declare no competing interests.
\end{abstract}




\begin{abstract}
Children acquire their first language while interacting with adults in a highly adaptive manner. While adaptation occurs at many linguistic levels such as syntax and speech complexity, semantic adaptation remains unclear due to the difficulty of efficient meaning extraction. In this study, we examine the adaptation of semantics with a computational approach based on distributional information. We show that adults, in their speech addressed to children, adapt their distributional semantics to that in the speech children produce. By analyzing semantic representations modeled from the Manchester corpus, a large longitudinal acquisition corpus of English, we find striking similarity of semantic development between child and child-directed speech, with a slight time lag in the latter. These findings provide strong evidence for the semantic adaptation in first language acquisition and suggest the important role of child-directed speech in semantic learning. Keywords: Child-directed speech, semantic adaptation, first language acquisition
\end{abstract}




\section{Adults adapt to child speech in semantic use}

One of the main challenges of language learning for children at an early age is to generalize word meaning. This challenge escalates when words lack explicit marking. For instance, lexical causatives that express causal meaning, such as teach (cause to learn) and raise (cause to rise), share no formal markers that can indicate the common meaning component of causation. Children therefore need to infer these commonalities of semantics from the linguistic cues in their surrounding speech. Currently, it is not yet clear how semantics is represented in child-directed speech (CDS) and how such representations are related to the semantic use in child speech (CS). To answer these questions, we investigate the development of semantic networks in both speech genres. To achieve this, we exploit a distributional learning algorithm which generates vector representations for semantics, and we construct semantic networks that best display the intricate relations between words. In particular, we pinpoint lexical causatives in our study. When understanding a lexical causative verb, children have to not only recognize the agent and the patient, but also disentangle the causing and caused events from the contexts. Therefore, semantics of lexical causatives is predominantly characterized by their distributional patterns of their surrounding linguistic units, making them a salient category for probing semantics. Our goal is to explore the dynamics of semantic learning and examine whether adaptation occurs between CDS and CS.

Despite the rapid growth of vocabulary size in the early years (Benedict, 1979, Goldfield \& Reznick, 1990; Nelson, 1973), children manage to establish a robust semantic system that allows error-free word choice and sentence formation (Ambridge \& Lieven, 2011; Bowerman, 1978; Mansfield, 1977). Here, the question remains what facilitating factors support semantic learning. Many studies focus on the importance of CDS, namely the speech addressed to children, for the development of children's linguistic abilities. (Hart \& Risley, 1995; Huttenlocher et al., 2002; Ma et al., 2011; Snow, 1972; Theakston et al., 2001). Indeed, it has been suggested that CDS is "fine-tuned" (Snow, 1989, Snow, 
2017; Sokolov, 1993) to adapt to CS at various linguistic levels (e.g., speech complexity by Kunert et al., 2011 and phonetics by Fernald, 1985, Kuhl et al., 1997; McRoberts and Best, 1997). The adjustment and adaptation at these levels have been claimed to facilitate children's linguistic development (Cross, 1979; Kuhl et al., 1997; Saint-Georges et al., 2013 Tamis-LeMonda et al., 2001). Yet, it is not clear whether such adaptation occurs at the level of semantics, nor do we know what factors facilitate semantic learning. A critical issue is that semantic abstraction is intricate and thus difficult to access (e.g., Asr et al., 2016). This so far has hindered in-depth investigations into semantic development in first language acquisition.

Previous studies have provided evidence for the adaptation of specific linguistic properties in CDS. For instance, early work by Furrow et al. (1979) has shown that speech complexity (e.g., utterance length and word types) in CDS significantly differs from adult-directed speech and the attributes of speech complexity in CDS can predict children's developmental growth. Sokolov (1993) further surveyed the local contingency in parent-child dyadic conversation, where they found that adult speech is closely-related to CS for selected word classes. Such correlation has recently been reassured with more synthesized measures by Kunert et al. (2011), where more controls for factors such as age were applied as well. Meanwhile, these studies also feature the correlation of syntactic structures between CDS and CS. Importantly, more recent work attempts to discover the directionality of this correlation and thereby clearly shows adults' adaptation to children. Examples include an analysis of syntactic descriptions in parent-child interaction by Dale and Spivey (2006), where the results suggested adults' coordination of syntactic use in their speech. In addition, Irvin et al. (2016) conducted a more systematic analysis on the dynamics between CDS and CS and found children's syntactic diversity to be the cause of that in adults' utterances. These results suggest that adults exhibit accommodation when the interlocutor is the child.

While similar measures are used to evaluate speech complexity (e.g., mean length of 
utterances) and syntactic complexity (e.g., grammatical categories such as word classes) across different studies, semantic adaptation has been evidenced by a much wider range of measures. These measures include indirect indicators borrowed from speech complexity (e.g., mean length of utterances, utterance types and word types as in Furrow et al., 1979, Roy et al., 2009). Other studies applied specially designed coding schemes to manually determine semantic similarity (e.g., Cross, 1979) and semantic complexity (e.g., sentence properties such as time and aspects in Barnes et al., 1983). On the other hand, coordination of semantics has also been implicated by the investigations into conversational cues, including repairs and repetitions (Clark \& Lappin, 2012; Demetras et al., 1986; Golinkoff, 1986, Morgenstern et al., 2013). Although the evidence seems abundant regarding semantic adaptation in CDS, most studies overlook the interplay between words with their intricate semantics in speech. Word meanings rarely stand in isolation from each other; rather, they often intertwine with each other in utterances and are hence difficult to disentangle. In the context of language acquisition, for instance, upon hearing a simple utterance as "He opens the window", children need to not only map "he" and "window" to their referents, but also establish the relation between them. More concretely, children have to assign the thematic roles of "agent" and "patient" to the two participants "he" and "window" respectively, so as to understand the causal meaning of "open", namely, "he opens the window, so the window opens". Such interplay between words can only be manifested by comprehensively examining the linguistic contexts in utterances. In other words, children's acquisition of semantics can be strongly associated with how information is distributed in contexts.

Rich evidence has been given on children's exploitation of contextual frames when inferring word meanings (Erickson \& Thiessen, 2015, Naigles, 1996, Pine \& Lieven, 1997; Theakston et al., 2001; Tomasello, 2003; Yuan et al., 2012). It has been shown that patterns can emerge from cross-situational contexts, thus facilitating lexical learning. This mechanism of statistical learning, often referred to as "distributional learning" (Pinker, 
1984), fundamentally allows for the learning of linguistic regularities. Co-occurring patterns are not only crucial to semantic understanding, but also vital for the acquisition of many linguistic aspects, from phonetics and phonology to semantics and syntax (Arnon, 2019; Bulf et al., 2011; Maye et al., 2008; Newport \& Aslin, 2004; Perfors et al., 2010. Saffran et al., 1996, Smith \& Yu, 2008, Yu \& Ballard, 2007). Besides, the experience of statistical learning in one linguistic aspect can even be extended to help with other learning tasks, such as word-object mapping (Estes et al., 2007) and meaning generalization (Lany \& Saffran, 2010). Hence, distributional learning can potentially be employed to build semantic representations that can best account for the embeddedness of semantics situated in linguistic contexts. This, however, has not been widely tested for naturalistic speech in parent-child interaction. In fact, compared to speech in experimental studies, naturalistic speech from large corpora includes richer cross-situational patterns on which distributional learning crucially relies. Therefore, distributional semantics extracted from naturalistic CDS and CS can substantially shed light on semantic learning.

Modern computational algorithms have rendered the possibility of comprehensive inference of distributional information to capture semantic regularities (e.g., Asr et al., 2016; Devlin et al., 2018, Hofmann, 1999: Mikolov et al., 2013). For instance, Mikolov et al. (2013) established models trained from contexts to efficiently represent word meanings. These models adjust themselves by optimally predicting a focus unit from its neighboring units, or vice versa, thus generating high-dimensional vectors that represent semantics. Semantic nuances thus can be identified by detecting the statistical regularities in their contexts. Though lacking concrete specifications of semantic abstraction, these vectors inherently reflect how distributions are shaped. Hence, verb categories that largely rely on distributional information in their inference can maximally highlight the efficacy of distributional learning algorithms. One category of this kind, which we use as a test case in our study, is lexical causatives that express causal meaning. A lexical causative, such as "open", "change", and "raise", describes a causal event where an agent acts on a patient, 
causing a change of state in the latter (Haspelmath, 2001, Shibatani, 2002), as in the above-mentioned sentence "He opens the window", where "he" causes "the window" to "open". This category possesses two distinctive features that promote distributional learning. First, lexical causatives lack explicit marking, therefore largely relying on contextual information to represent the causative meaning. Second, unlike non-causatives such as "think" and "rise", a lexical causative establishes a prominent link between subject and object, which is associated with many intrinsic features (e.g., animacy) of participants in the verbal event (Arka, 1993 Comrie \& Polinsky, 1993; Dixon, 2000; Shibatani, 2002). Hence, semantic nuances of co-occurring words are especially important for the inference of lexical causatives. Moreover, it has been suggested that lexical causatives can be successfully discriminated from non-causatives with the help of distributional learning algorithms (You et al., 2020). We can thus pinpoint the distributional semantics of lexical causatives to examine the semantic coordination between CDS and CS.

Here, we target a group of prototypical lexical causatives that commonly exist in human language (Haspelmath, 1993). To test the generalization of causative semantics, it is beneficial to evaluate to what extent word meanings are connected to each other. This is most realizable with the assistance of a complex network, which has been proposed and verified to exhibit word associations (Dorogovtsev \& Mendes, 2001; Senekal \& Kotzé, 2017 Steyvers \& Tenenbaum, 2005 Utsumi, 2015). Particularly, Utsumi (2015) and Chen (2020) have recently shown that complex networks built on distributional semantics are able to reflect human lexical knowledge. The causative semantic network can accordingly be expanded with associated words discovered by models of distributional semantics and reflect causative semantics of the most relevance. We thereby employ a computational approach that combines both distributional semantics and complex networks to investigate the development of semantics in first language acquisition. More specifically, in this study, we exploit word embeddings (Mikolov et al., 2013). Word embeddings systematically infer from co-occurrence patterns, and establish networks that center on causative semantics. 
We examine how the semantics of this prominent category is generalized in both CDS and CS. We explore what semantic information children longitudinally receive from CDS and how they in turn shape semantics their own production. Concretely, we aim to answer two questions regarding the role of CDS for semantic learning: (1) How do the dynamics of semantic representations develop in CDS and CS over time? (2) Does adaptation occur between CDS and CS in terms of semantic learning?

\section{Methods}

\section{Data}

Our data comes from the Manchester Corpus (Theakston et al., 2001), a longitudinal corpus of language acquisition of English. It consists of naturalistic speech of 12 children and their caregivers, with the starting age ranging from 20 to 24 months with recordings up to approximately 36 months (see details in Supplementary Materials Table S1). Recordings took place every 3 weeks. The corpus is fully lemmatized and annotated for part-of-speech tags. In order to obtain meaningful semantic representations from distributional information (Lai et al., 2016), we merged sessions with a sliding-window approach (Henzinger et al., 2009; Irvin et al., 2016) to create a larger data size. With the window size 2 , sessions of every two consecutive months in age were merged with a monthly interval and the merged data was marked with the age of the last session (e.g., for the age range 25-28 months, three merged data sets, i.e. speech of months $25-26$, months 26-27, and months $27-28$, were created, and were marked as " 26 ", " 27 " and " 28 " months in age respectively). For each child, this merging process was applied in CS and CDS separately. This sliding-window approach also ensured that the vocabulary sizes across different ages were comparable within each child (see Supplementary Material Fig. S3 and Fig. S4). We did not opt for a larger merging window, in that sufficient data points were required for time-series analysis to reveal the development over time. 
Input layer

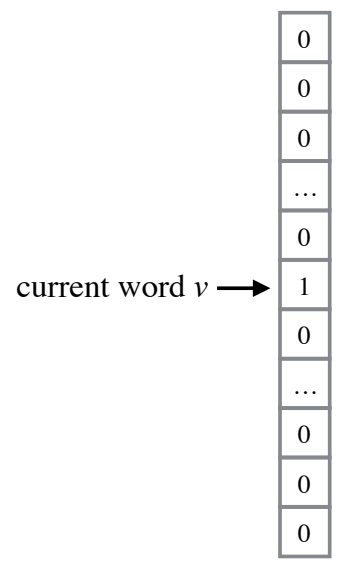

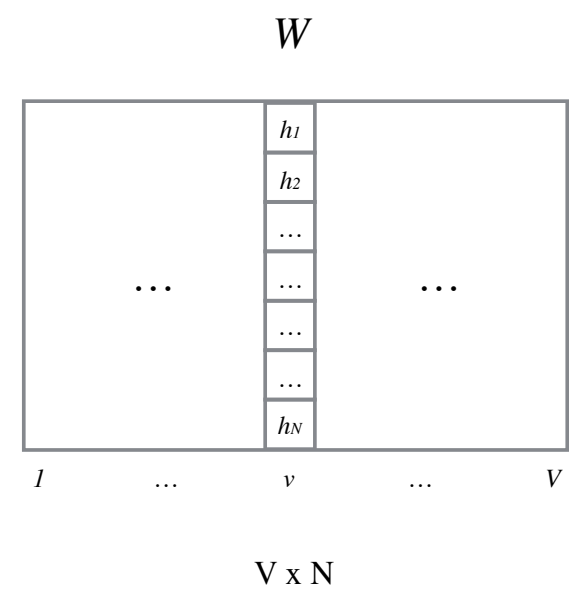

Hidden layer

Output layer

$W^{\prime}$

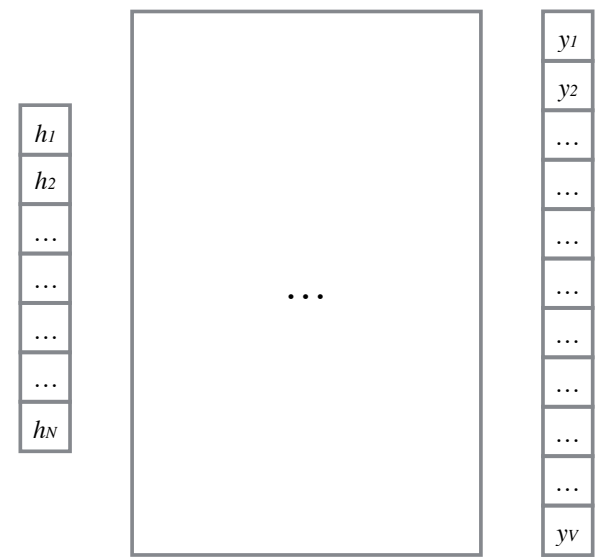

$1 \times \mathrm{N}$

$1 \times \mathrm{V}$

\section{Figure 1}

$1 \times \mathrm{V}$

$\mathrm{N} \times \mathrm{V}$

The network of skip-gram word embeddings. W denotes weight matrix that contains the information of vectors, and $W$ ' stands for the weight matrix that computes the relations between words and the contexts. The output layer is later transformed into a vector $\left(y_{1}, y_{2}, \ldots, y_{V}\right)$ of probabilities that determine the predicted contexts. During the training phase, both matrices are updated based on the feedback from the the predictions produced for each training instance.

\section{Generating semantic representations}

We employed skip-gram word embeddings (Mikolov et al., 2013) to generate vectors that represent the semantics of words. Skip-gram word embeddings is a Word2Vec algorithm based on co-occurrence patterns. The algorithm uses a neural network to predict the contextual words within a symmetrical window from every word in a corpus (see Fig. 11).

The neural network is updated after the training of each word-context pair by comparing the predicted contexts with the true contexts, and eventually the weight matrix before the hidden layer in the network contains the vectors of all words in the vocabulary. Skip-gram word embeddings thereby builds associations between words based on 
distributional information, and, as a result, words are projected to vectors in a high-dimensional space. The generated vectors have proven efficient in capturing semantic regularities (Mikolov et al., 2013). Particularly, it is shown that word embeddings trained

on adjacent raw contexts in CDS can facilitate causative discrimination (You et al., 2020). This might be accounted for by the compact structures in utterances that are shorter on average. Hence, we applied the smallest context range, namely one word on each side, in the training of word vectors. We allowed vectors generated for all words with a minimum count of 2 occurrences, so that they inferred the semantics from at least two varied contextual environments. The dimension of vectors ( $N$ in Fig. 1) was set to 200 and each model iterates 100 times over the input data. For every merged data set, we developed one corresponding embeddings model with such settings.

\section{Constructing a causative semantic network}

To construct a causative network that reflects the dynamics of distributional information, we capitalized on the word vectors to find the most relevant words in the domain of causative semantics. Prototypical lexical causatives that commonly exist across human languages, as determined by typological research, were selected to represent the central semantics (Haspelmath, 1993).

- Causatives (23): begin, boil, break, burn, change, close, destroy, dry, fill, finish, freeze, gather, kill, lose, melt, open, raise, roll, sink, spread, stop, teach, turn

We extended our search of causative relevance to the most similar words of these prototypes in the embeddings models. That is, in each embeddings model, we collected available prototypes (i.e. prototypical causatives that occur at least twice) with their $N$ most similar words based on cosine distance. Here, $N$ was determined by a fixed ratio of 50 to the vocabulary size, with the ceiling number taken. While a large ratio controls the impact of vocabulary size, increasing this ratio could potentially hinder us from finding 
enough words of semantic relevance. We thus employed approximately half of the standard deviation of vocabulary sizes in both genres (CDS: $S D=122.5$; CS: $S D=93.8$ ).

These pairs of similar words were subsequently connected with edges to form a graph, with the words as vertices. Additionally, we removed the vertices with degree 1, namely, words with only one similar word in the entire graph. These words do not bridge any vertices in the graph and are thus of the least relevance in terms of causative semantics. We thereby transferred the semantic representations in each word embeddings model to a graph of causative semantic network.

\section{Operationalization of semantic complexity and baseline}

We operationalized the complexity of causative semantics as the number of edges of the largest connected component (LCC) in the causative network. The LCC identifies the sub-graph with the largest number of vertices, and therefore diagnoses the most

concentrated component in a graph (Baeza-Yates \& Tiberi, 2007; Morais et al., 2013). This sub-graph can indicate how lexicons of causative relevance are connected. To account for both the scale and the connectedness of the LCC, we measured the number of edges, namely the number of connections between words in the most relevant network that represents causative semantics. This can suggest how densely the causative-related words are aggregated and to what degree causative semantics is generalized.

Meanwhile, we considered a baseline for each word embeddings model. We randomly selected verbs of the same number as that of the available prototypical causatives in the respective data set, and constructed a random graphs using the same procedure as the causative network. 1,000 random graphs as such were built, and we examined each graph to acquire the median of the distribution of edges in the LCC (we chose median over average since the distribution could be highly skewed). This baseline serves as an indication of overall semantic complexity at the current stage. By measuring the above-baseline performance, we can infer how much complexity a causative network has 
gained above the overall level. Moreover, this baseline further eliminates the effect by different vocabulary sizes across ages. As a last step, we divided the above-baseline performance by the number of available prototypical causatives, so as to show how much the causative semantics is extended by each causative.

\section{Analyses}

In Analysis 1, we performed segmented regression to capture the dynamics of the causative network in CS and CDS respectively, where network complexity was regressed on age. We aimed to examine breakpoints and trends of development of causative semantics in each speech genre, and thus evaluated the adaptation between adults and children. Our primary focus was on the overall development by all recorded children other than the individual performances. Hence, we employed Bayesian hierarchical regression, where individual children were treated as a group factor (see Fig. S9 to Fig. S17 for the additional examination of random slopes at individual levels).

The segmented regression was implemented with grid search (Lerman, 1980). We preserved an age span of at least 3 months for each segment, and limited the number of breakpoints to 2 to avoid over-complication. To compare models with different breakpoints, we performed pseudo Bayesian model averaging (pseudo-BMA+, which is stabilized with the Bayesian bootstrap) (Yao et al., 2018) to check the model weights of all candidate models. Model averaging with BMA + has proven effective in eliminating selection-based bias with a large number of candidate models, and therefore can serve as the method for model selection (e.g., Harrison et al., 2019; Höge et al., 2020). We report the models that leverage noticeable weights in the results section.

In Analysis 2, we regressed the network difference between CS and CDS on age with the same approach as in Analysis 1. This was to survey how the gap between the two speech genres changes over time, as compared with their respective development. 

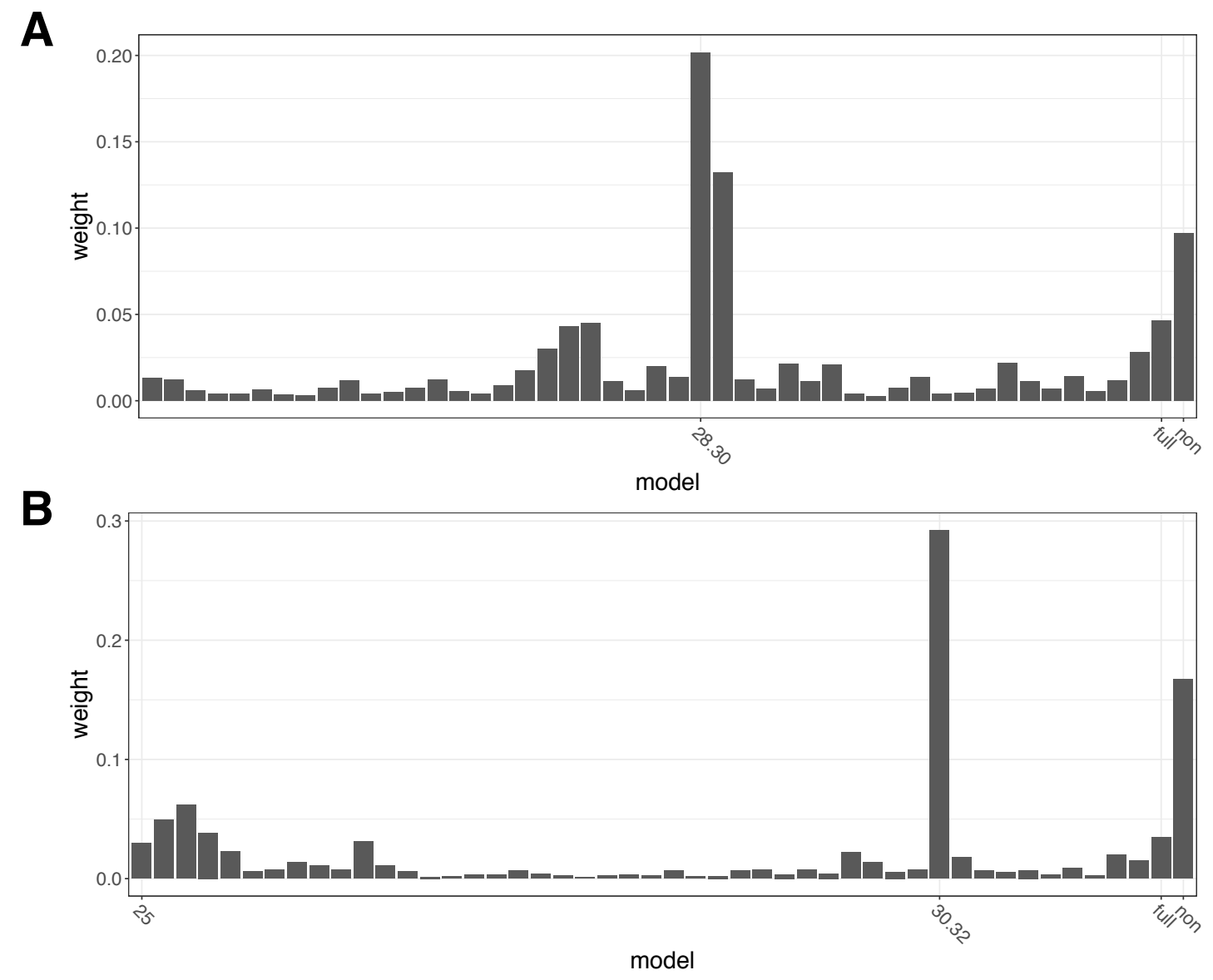

\section{Figure 2}

Model weights using model averaging with pseudo-BMA+. Models with two breakpoints are denoted by the label format "point1.point2". (A) candidate models for CS; (B) candidate models for $C D S$

\section{Results}

\section{Analysis 1}

We first investigated the development of complexity of causative semantics in CS. The result of model averaging shows that the model with the breakpoints at the months 28 and 30 leverages the most weight among all candidate models (see Fig. 2A $\mathrm{A}$; for posterior predictive checking, see Fig. S5 to Fig. S8).

Fig. 33A depicts the predicted values of this model. There is a significant increase of 

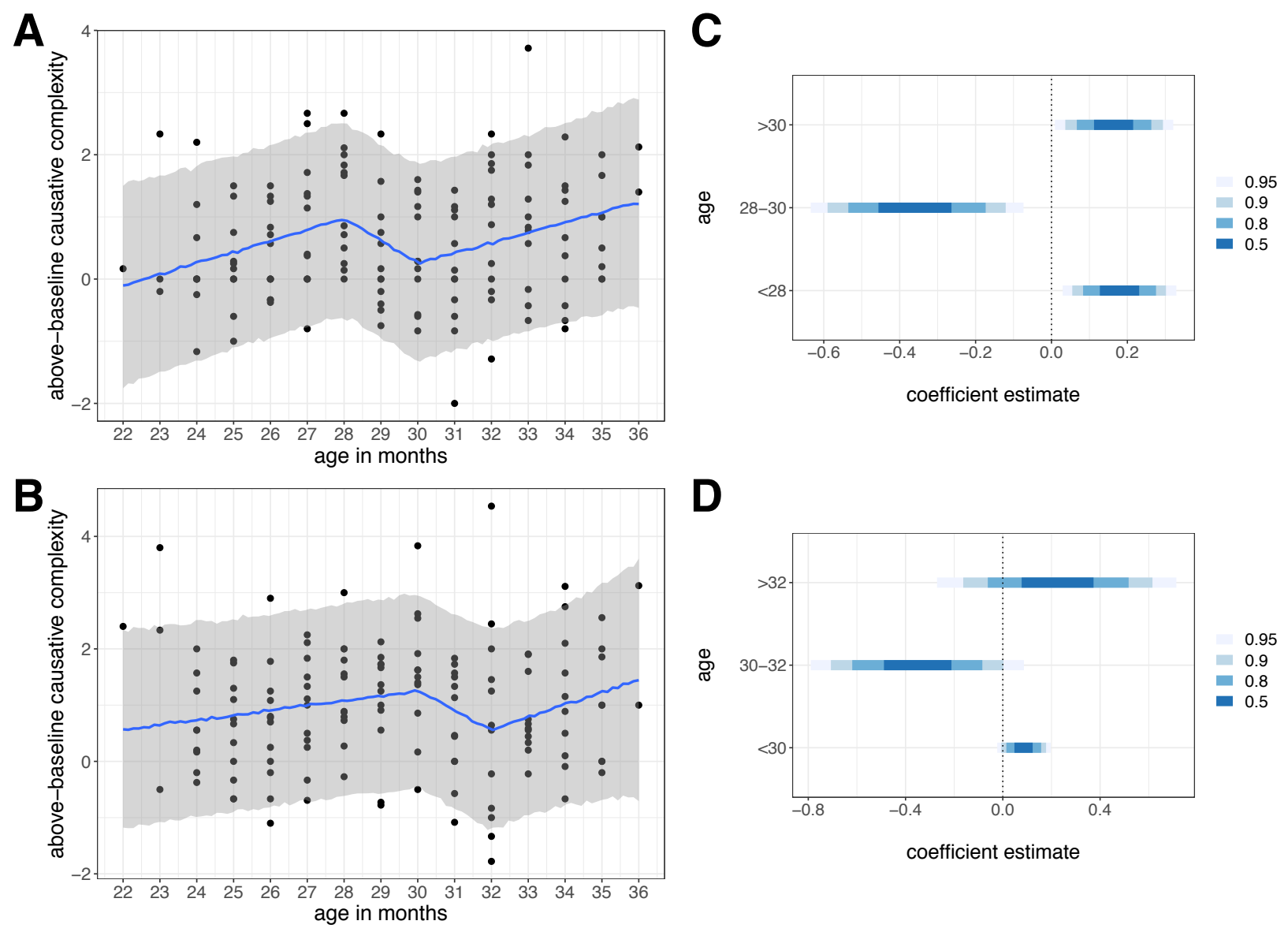

\section{Figure 3}

Summary of the model that leverages the most weight in each genre. Predicted values with shaded 90\% quantile-based credible interval, with observed data points plotted: (A) CS; (B) CDS. Quantile-based credible interval for coefficients of three segments, respectively: (C) $C S ;(D) C D S$.

causative complexity (see posterior distribution of coefficients in Fig. $3 \mathrm{~B}$ ) both before 28 months old (90\% CI: $[0.055,0.302])$ and after 30 months old (90\% CI: [0.036, 0.294]). However, the complexity drops significantly between the months 28 and 30 (90\% CI: $[-0.590,-0.121])$. This suggests that despite a general uptrend over time regarding children's development of causative semantics, stagnation of development occurs in the period between months 28 and 30 . 
CDS shows striking similarity in development compared to CS. Model averaging with pseudo-BMA + substantially favors the model with breakpoints at the age of 30 and 32 months (see Fig. 2B). As displayed in Fig. 3D, the trend before 30 months is shown to be positive (93.9\% of the posterior estimates are greater than 0$)$, whereas the causative complexity sharply declines from 30 months to 32 months $(94.9 \%$ of the posterior estimates are below 0; see Fig. 3D). This period of decrease occurs slightly later in CDS than that in CS, with a 2-month delay. This indicates that adults might adjust their speech after observing the reduced performance of semantic representations by children. Similarly to the resumption of development in CS, causative complexity in CDS again increases after the age of 32 months.

\section{Analysis 2}

The model without age as a factor outweighs all the other models in model averaging (see Fig. 4A). This indicates that the gap between CS and CDS does not exhibit clear change over time. Nonetheless, when breakpoints fall within the range from 27 to 30 months, models leverage noticeable weights compared to the other candidate models. In particular, we examined the most prominent model with the breakpoints at 27 and 30 months. As shown in Fig. $4 \mathrm{~B}$, CS keeps approaching CDS before 27 months $(94.4 \%$ of the posterior estimates are below 0), at which point the gap in between starts to widen (97.2\% of the posterior estimates are greater than 0 ). The uptrend is halted at the age of 30 months (94.9\% of the posterior estimates are below 0$)$, which parallels the dynamics in CDS. This indicates that the adjustment in CDS might be triggered by an observed enlarged gap with CS. More importantly, the facilitation of such adjustment manifests itself in the last stage, where children restart to assimilate to adults' semantic complexity.

\section{Discussion}

Overall, our results show that children do not develop their causative semantics monotonically over time, and adults accordingly adapt their speech to ease the acquisition 


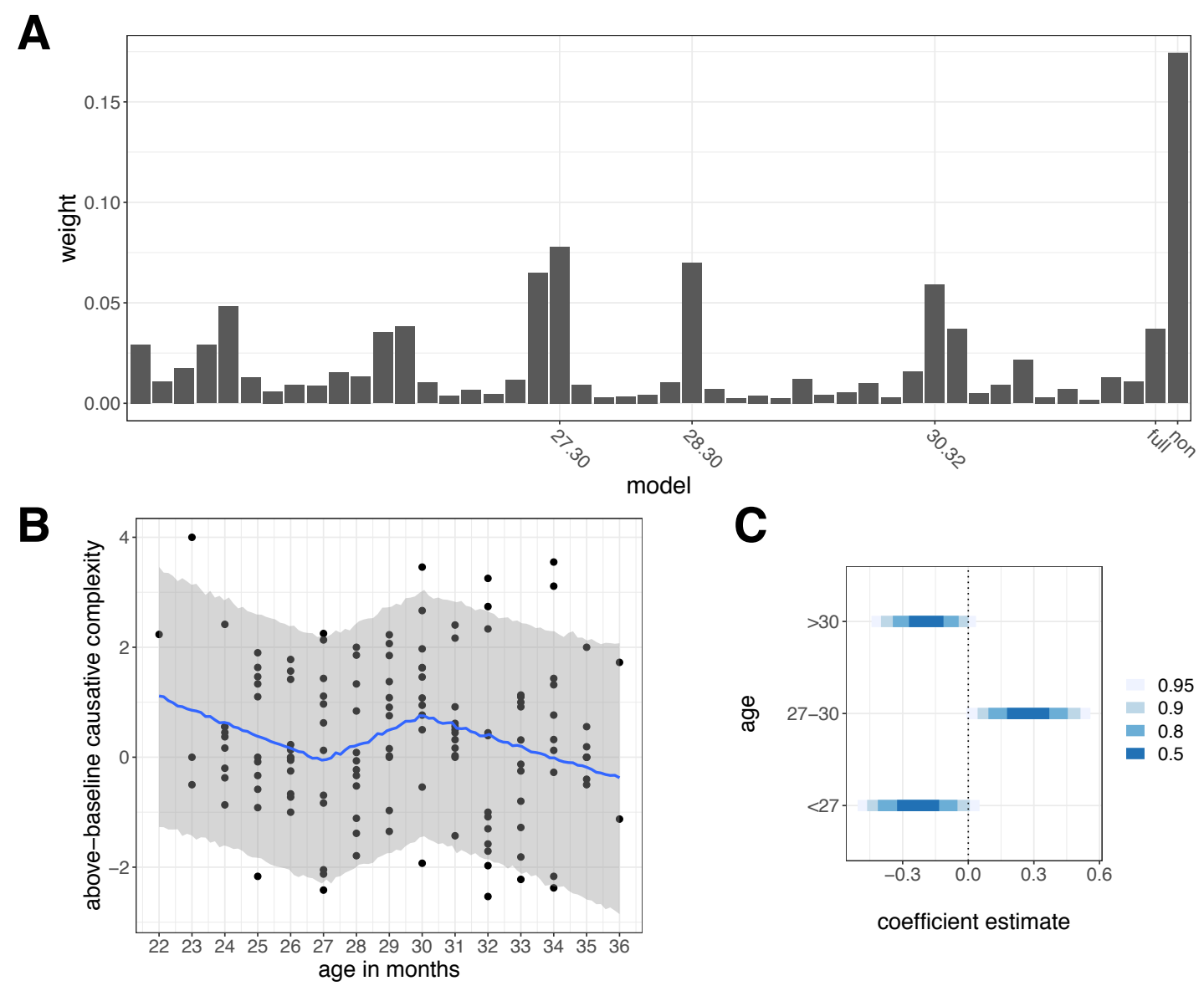

\section{Figure 4}

Summary of Analysis 2: segmented regression of genre difference (CDS - CS) on age. (A) The model with breakpoints at 27 and 30 months weighs the second to the model without age as a predictor. (B) Predicted values (with shaded 90\% quantile-based credible interval) show that the gap between CS and CDS boosts between 27 and 30 months, while declining before 27 months and after 30 months. (C) Quantile-based credible interval for each coefficient. The effects of all three periods are substantial.

of word meanings, which is in accordance with the fine-tuning theory. On the one hand, while steadily increasing their complexity in expressing causatives, children show a sharp decline of performance around the age of 28-30 months. We suggest that the rapid growth of vocabulary size and utterance complexity (e.g., mean length of utterances, i.e. MLU) 
might play a role in this change. This rapid growth could potentially perplex the semantic categorization and thus lead to temporary performance drop. For instance, Dapretto and Bjork (2000) found a major burst of naming errors, especially categorical errors, when lexical development starts to become prominent. Further, as the MLU increases (see Fig. S1 and Fig. S2 in Supplementary materials), words are allowed to be situated in more variant contexts, which could increase the difficulty of categorization. For example, Scarborough (1990) showed that, for children from 2 to 4 years old, the syntactic complexity is strongly correlated with MLU at younger ages. In addition, linguistic development is suggested to closely interact with many cognitive abilities, such as lexical memory and the ability of categorization, and the asynchronicity between these two developments could cause the idiosyncrasies in language acquisition (e.g., Gopnik and Meltzoff, 1987; Woodward et al., 1994).

On the other hand, CDS exhibits striking resemblance to CS in terms of the development of causative complexity. In particular, the period of reduced complexity in CDS begins immediately when CS ends its decline. It could be suggested that the change in CS triggers the adjustment in CDS. Adults might observe the difficulty of connecting the semantics across a large number of words for children at the earlier stage, and subsequently express words in more distinctive patterns. This helps children retain efficient distributional cues that assist with their understanding of word clusters on a smaller scale, thus resuming their generalization of semantics at a later stage. In addition, the smaller gap between CS and CDS during this period points to the facilitation of such adaptation in the learning of causative semantics. Nevertheless, it should be noted that age in general does not significantly predict the gap between two genres. This indicates that the adaptation from CDS to CS does not substantially enhance the performance of the learning of word meanings; rather, it might merely prevent CS from diverging from the adult-like semantic complexity. However, considering the ceiling of semantic complexity that adults' speech will soon reach, children could approach the adults' level with a slight delay of 
several months.

Lastly, the adaptation in our findings also resonates with the importance of distributional cues in first language acquisition. The semantic representations generated via distributional learning manage to render rather similar developments in CS and CDS. Therefore, distributional cues from simple linear strings without structural abstraction might prevail in word learning, especially verb learning, at an early stage, and should hence receive more attention in future research of language acquisition.

We conclude that adults adapt to children in their semantic learning. With a novel computational approach based on distributional learning, we investigate semantic representations in both CDS and CS by maximally attending to their cross-situational patterns. We hence tackle the traditional obstacle of holistically accessing semantics in surveying language acquisition. Most importantly, we highlight the important role of CDS in children's semantic development, adding yet another level to the multifaceted facilitation of CDS for first language acquisition.

\section{Acknowledgments}

The results of this research are part of the project "The role of causality in early verb learning: language-specific factors vs. universal strategies" (Swiss NSF Grant agreement No. 100015_169712, Stoll, Daum), the project “Acquisition processes in maximally diverse languages: Min(ding) the ambient languages (ACQDIV)" that has received funding from the European Research Council (ERC) under the European Union's Seventh Framework Programme FP7-2007-2013 (Grant agreement No. 615988; Stoll), and the NCCR Evolving Language, Swiss National Science Foundation Agreement No. 51NF40_180888 (Stoll, Daum). 


\section{References}

Ambridge, B., \& Lieven, E. V. M. (2011). Child language acquisition: Contrasting theoretical approaches. Cambridge University Press.

Arka, I. W. (1993). Morpholexical aspects of the -kan causative in indonesian (Master's thesis). University of Sydney.

Arnon, I. (2019). Statistical learning, implicit learning, and first language acquisition: A critical evaluation of two developmental predictions. Topics in Cognitive Science.

Asr, F. T., Willits, J., \& Jones, M. N. (2016). Comparing predictive and co-occurrence based models of lexical semantics trained on child-directed speech. CogSci.

Baeza-Yates, R., \& Tiberi, A. (2007). Extracting semantic relations from query logs. Proceedings of the 13th ACM SIGKDD international conference on Knowledge discovery and data mining, 76-85.

Barnes, S., Gutfreund, M., Satterly, D., \& Wells, G. (1983). Characteristics of adult speech which predict children's language development. Journal of child language, 10(1), $65-84$.

Benedict, H. (1979). Early lexical development: Comprehension and production. Journal of child language, 6(2), 183-200.

Bowerman, M. (1978). Systematizing semantic knowledge: Changes over time in the child's organization of word meaning. Child Development, 977-987.

Bulf, H., Johnson, S. P., \& Valenza, E. (2011). Visual statistical learning in the newborn infant. Cognition, 121(1), 127-132.

Chen, A. C.-H. (2020). Words, constructions and corpora: Network representations of constructional semantics for mandarin space particles. Corpus Linguistics and Linguistic Theory, 1 (ahead-of-print).

Clark, A., \& Lappin, S. (2012). Complexity in language acquisition. Topics in Cognitive Sciences, 5(1), 89-110. 
Comrie, B., \& Polinsky, M. (1993). Causatives and transitivity (Vol. 23). John Benjamins Publishing.

Cross, T. G. (1979). Mothers' speech adjustments and child language learning: Some methodological considerations. Language Sciences, 1(1), 3-25.

Dale, R., \& Spivey, M. J. (2006). Unraveling the dyad: Using recurrence analysis to explore patterns of syntactic coordination between children and caregivers in conversation. Language Learning, 56(3), 391-430.

Dapretto, M., \& Bjork, E. L. (2000). The development of word retrieval abilities in the second year and its relation to early vocabulary growth. Child development, 71(3), $635-648$.

Demetras, M. J., Post, K. N., \& Snow, C. E. (1986). Feedback to first language learners: The role of repetitions and clarification questions. Journal of child language, 13(2), 275-292.

Devlin, J., Chang, M.-W., Lee, K., \& Toutanova, K. (2018). Bert: Pre-training of deep bidirectional transformers for language understanding. arXiv preprint arXiv:1810.04805.

Dixon, R. M. W. (2000). A typology of causatives: Form, syntax and meaning. Changing valency: Case studies in transitivity (pp. 30-83). Cambridge University Press, Cambridge.

Dorogovtsev, S. N., \& Mendes, J. F. F. (2001). Language as an evolving word web. Proceedings of the Royal Society of London. Series B: Biological Sciences, 268(1485), 2603-2606.

Erickson, L. C., \& Thiessen, E. D. (2015). Statistical learning of language: Theory, validity, and predictions of a statistical learning account of language acquisition. Developmental Review, 37, 66-108. 
Estes, K. G., Evans, J. L., Alibali, M. W., \& Saffran, J. R. (2007). Can infants map meaning to newly segmented words? statistical segmentation and word learning. Psychological science, 18(3), 254-260.

Fernald, A. (1985). Four-month-old infants prefer to listen to motherese. Infant behavior and development, 8(2), 181-195.

Furrow, D., Nelson, K., \& Benedict, H. (1979). Mothers' speech to children and syntactic development: Some simple relationships. Journal of Child Language, 6(03), 423-442.

Goldfield, B. A., \& Reznick, J. S. (1990). Early lexical acquisition: Rate, content, and the vocabulary spurt. Journal of Child Language, 17, 171-183.

Golinkoff, R. M. (1986).

i beg your pardon?': The preverbal negotiation of failed messages. Journal of child language, 13(3), 455-476.

Gopnik, A., \& Meltzoff, A. (1987). The development of categorization in the second year and its relation to other cognitive and linguistic developments. Child development, $1523-1531$.

Harrison, J. U., Parton, R. M., Davis, I., \& Baker, R. E. (2019). Testing models of mrna localization reveals robustness regulated by reducing transport between cells. Biophysical Journal, 117(11), 2154-2165.

Hart, B., \& Risley, T. R. (1995). Meaningful differences in the everyday experience of young american children. Paul Brookes Publishing.

Haspelmath, M. (1993). More on the typology of inchoative/causative verb alternations. In B. Comrie \& M. Polinsky (Eds.), Causatives and transitivity (pp. 87-121). John Benjamins.

Haspelmath, M. (2001). The european linguistic area: Standard average european. In M. Haspelmath, E. König, W. Oesterreicher, \& W. Raible (Eds.), Language typology and language universals: An international handbook (pp. 1492-1510). Mouton de Gruyter. 
Henzinger, T. A., Mateescu, M., \& Wolf, V. (2009). Sliding window abstraction for infinite markov chains. International Conference on Computer Aided Verification, 337-352.

Hofmann, T. (1999). Probabilistic latent semantic analysis. Proceedings of the Fifteenth conference on Uncertainty in artificial intelligence, 289-296.

Höge, M., Guthke, A., \& Nowak, W. (2020). Bayesian model weighting: The many faces of model averaging. Water, $12(2), 309$.

Huttenlocher, J., Vasilyeva, M., Cymerman, E., \& Levine, S. (2002). Language input and child syntax. Cognitive Psychology, 45, 337-374.

Irvin, J., Spokoyny, D., \& del Prado Martin, F. M. (2016). Dynamical systems modeling of the child-mother dyad: Causality between child-directed language complexity and language development. CogSci.

Kuhl, P. K., Andruski, J. E., Chistovich, I. A., Chistovich, L. A., Kozhevnikova, E. V., Ryskina, V. L., Stolyarova, E. I., Sundberg, U., \& Lacerda, F. (1997). Cross-language analysis of phonetic units in language addressed to infants. Science, 2777(5326), 684-686.

Kunert, R., Fernández, R., Zuidema, W., et al. (2011). Adaptation in child directed speech: Evidence from corpora. Proc. SemDial, 112119.

Lai, S., Liu, K., He, S., \& Zhao, J. (2016). How to generate a good word embedding. IEEE Intelligent Systems, 31(6), 5-14.

Lany, J., \& Saffran, J. R. (2010). From statistics to meaning: Infants' acquisition of lexical categories. Psychological science, 21(2), 284-291.

Lerman, P. (1980). Fitting segmented regression models by grid search. Journal of the Royal Statistical Society: Series C (Applied Statistics), 29(1), 77-84.

Ma, W., Golinkoff, R. M., Houston, D. M., \& Hirsh-Pasek, K. (2011). Word learning in infant-and adult-directed speech. Language Learning and Development, 7(3), $185-201$. 
Mansfield, A. F. (1977). Semantic organization in the young child: Evidence for the development of semantic feature systems. Journal of Experimental Child Psychology, $23(1), 57-77$.

Maye, J., Weiss, D. J., \& Aslin, R. N. (2008). Statistical phonetic learning in infants: Facilitation and feature generalization. Developmental Science, 11(1), 122-134.

McRoberts, G. W., \& Best, C. T. (1997). Accommodation in mean f0 during mother-infant and father-infant vocal interactions: A longitudinal case study. Journal of Child Language, 24(3), 719-736. https://doi.org/10.1017/S030500099700322X

Mikolov, T., Chen, K., Corrado, G., \& Dean, J. (2013). Efficient estimation of word representations in vector space. Proceedings of ICLR Workshops Track.

Morais, A. S., Olsson, H., \& Schooler, L. J. (2013). Mapping the structure of semantic memory. Cognitive science, 37(1), 125-145.

Morgenstern, A., Leroy-Collombel, M., \& Caët, S. (2013). Self-and other-repairs in child-adult interaction at the intersection of pragmatic abilities and language acquisition. Journal of pragmatics, 56, 151-167.

Naigles, L. R. (1996). The use of multiple frames in verb learning via syntactic bootstrapping. Cognition, 58(2), 221-251.

Nelson, K. (1973). Structure and strategy in learning to talk (Vol. 38). Blackwell.

Newport, E. L., \& Aslin, R. N. (2004). Learning at a distance i. statistical learning of non-adjacent dependencies. Cognitive Psychology, 48(2), 127-162.

Perfors, A., Tenenbaum, J., \& Wonnacott, E. (2010). Variability, negative evidence, and the acquisition of verb argument constructions. Journal of Child Language, 37(03), $607-642$.

Pine, J. M., \& Lieven, E. V. (1997). Slot and frame patterns and the development of the determiner category. Applied Psycholinguistics, 18, 123-138.

Pinker, S. (1984). Language learnability and language development. Harvard University Press. 
Roy, B. C., Frank, M. C., \& Roy, D. (2009). Exploring word learning in a high-density longitudinal corpus.

Saffran, J. R., Aslin, R. N., \& Newport, E. L. (1996). Statistical learning by 8-month-old infants. Science, 274, 1926-1928.

Saint-Georges, C., Chetouani, M., Cassel, R., Apicella, F., Mahdhaoui, A., Muratori, F., Laznik, M.-C., \& Cohen, D. (2013). Motherese in interaction: At the cross-road of emotion and cognition?(a systematic review). PloS One, 8(10), e78103.

Scarborough, H. S. (1990). Index of productive syntax. Applied psycholinguistics, 11(1), $1-22$.

Senekal, B., \& Kotzé, E. (2017). Die statistiese eienskappe van geskrewe afrikaans as'n komplekse netwerk: Geesteswetenskappe. Litnet Akademies:'n Joernaal vir die Geesteswetenskappe, Natuurwetenskappe, Regte en Godsdienswetenskappe, 14(1), $27-59$.

Shibatani, M. (2002). The grammar of causation and interpersonal manipulation (Vol. 48). John Benjamins Publishing.

Smith, L. B., \& Yu, C. (2008). Infants rapidly learn word-referent mappings via cross-situational statistics. Cognition, 106(3), 1558-1568.

Snow, C. E. (1989). Understanding social interaction and language acquisition: Sentences are not enough. In M. H. Bornstein \& J. S. Bruner (Eds.), Interaction in human development (pp. 83-103). Lawrence Erlbaum Associates.

Snow, C. E. (1972). Mothers' speech to children learning language. Child development, $549-565$.

Snow, C. E. (2017). Issues in the study of input: Finetuning, universality, individual and developmental differences, and necessary causes. The handbook of child language, $179-193$.

Sokolov, J. L. (1993). A local contingency analysis of the fine-tuning hypothesis. Developmental psychology, 29(6), 1008. 
Steyvers, M., \& Tenenbaum, J. B. (2005). The large-scale structure of semantic networks: Statistical analyses and a model of semantic growth. Cognitive science, 29(1), 41-78.

Tamis-LeMonda, C. S., Bornstein, M. H., \& Baumwell, L. (2001). Maternal responsiveness and children's achievement of language milestones. Child Development, 72(3), $748-767$.

Theakston, A. L., Lieven, E. V. M., Pine, J. M., \& Rowland, C. F. (2001). The role of performance limitations in the acquisition of verb-argument structure: An alternative account. Journal of Child Language, 28, 127-152.

Tomasello, M. (2003). Constructing a language: A usage-based theory of language acquisition. Harvard University Press.

Utsumi, A. (2015). A complex network approach to distributional semantic models. PloS one, $10(8)$, e0136277.

Woodward, A. L., Markman, E. M., \& Fitzsimmons, C. M. (1994). Rapid word learning in 13-and 18-month-olds. Developmental psychology, 30(4), 553.

Yao, Y., Vehtari, A., Simpson, D., Gelman, A., et al. (2018). Using stacking to average bayesian predictive distributions (with discussion). Bayesian Analysis, 13(3), 917-1007.

You, G., Bickel, B., Daum, M. M., \& Stoll, S. (2020). Child-directed speech is statistically optimized for meaning extraction. https://doi.org/10.31234/osf.io/zmktd

Yu, C., \& Ballard, D. H. (2007). A unified model of early word learning: Integrating statistical and social cues. Neurocomputing, 70(13-15), 2149-2165.

Yuan, S., Fisher, C., \& Snedeker, J. (2012). Counting the nouns: Simple structural cues to verb meaning. Child Development, 83(4), 1382-1399. 


\section{Supplementary materials}

Codes and data can be access here.

\section{Corpus details}

\subsection{Age span and data size}

\section{Table S1}

Age span and data size for each child in the Manchester corpus

\begin{tabular}{l|l|c|c|c|l}
\hline \multirow{2}{*}{ Child } & Age span & \multicolumn{2}{|c|}{ Child-directed speech } & \multicolumn{2}{c}{ Child speech } \\
\cline { 2 - 6 } & Utterances & Words & Utterances & Words \\
\hline Child 1 & $1 ; 10.7-2 ; 9.10$ & 37,554 & 152,940 & 20,701 & 48,831 \\
\hline Child 2 & $1 ; 11.12-2 ; 10.28$ & 36,155 & 210,191 & 17,577 & 47,919 \\
\hline Child 3 & $2 ; 0.7-2 ; 11.15$ & 27,988 & 111,304 & 24,399 & 58,489 \\
\hline Child 4 & $1 ; 8.22-2 ; 8.15$ & 23,026 & 96,594 & 25,567 & 68,914 \\
\hline Child 5 & $1 ; 10.25-2 ; 10.16$ & 37,971 & 145,981 & 21,914 & 46,949 \\
\hline Child 6 & $1 ; 11.27-2 ; 11.12$ & 29,272 & 11,8162 & 17,277 & 42,751 \\
\hline Child 7 & $1 ; 11.1-2 ; 10.11$ & 33,428 & 131,666 & 18,755 & 44,285 \\
\hline Child 8 & $1 ; 11.15-2 ; 10.24$ & 21,201 & 92,123 & 13,763 & 30,478 \\
\hline Child 9 & $1 ; 11.9-2 ; 10.18$ & 20,900 & 89,577 & 16,660 & 41,329 \\
\hline Child 10 & $2 ; 0.25-3 ; 0.10$ & 33,143 & 141,491 & 17,771 & 32,897 \\
\hline Child 11 & $1 ; 11.15-2 ; 11.21$ & 36,786 & 151,148 & 20,824 & 40,760 \\
\hline Child 12 & $1 ; 10.6-2 ; 9.20$ & 25,334 & 129,393 & 17,179 & 49,565 \\
\hline
\end{tabular}

\subsection{Mean length of utterances (MLU)}

Fig. S1 and Fig. S2 below show the development of MLU in both child and

child-directed speech respectively for each child in the corpus. While MLU remains 


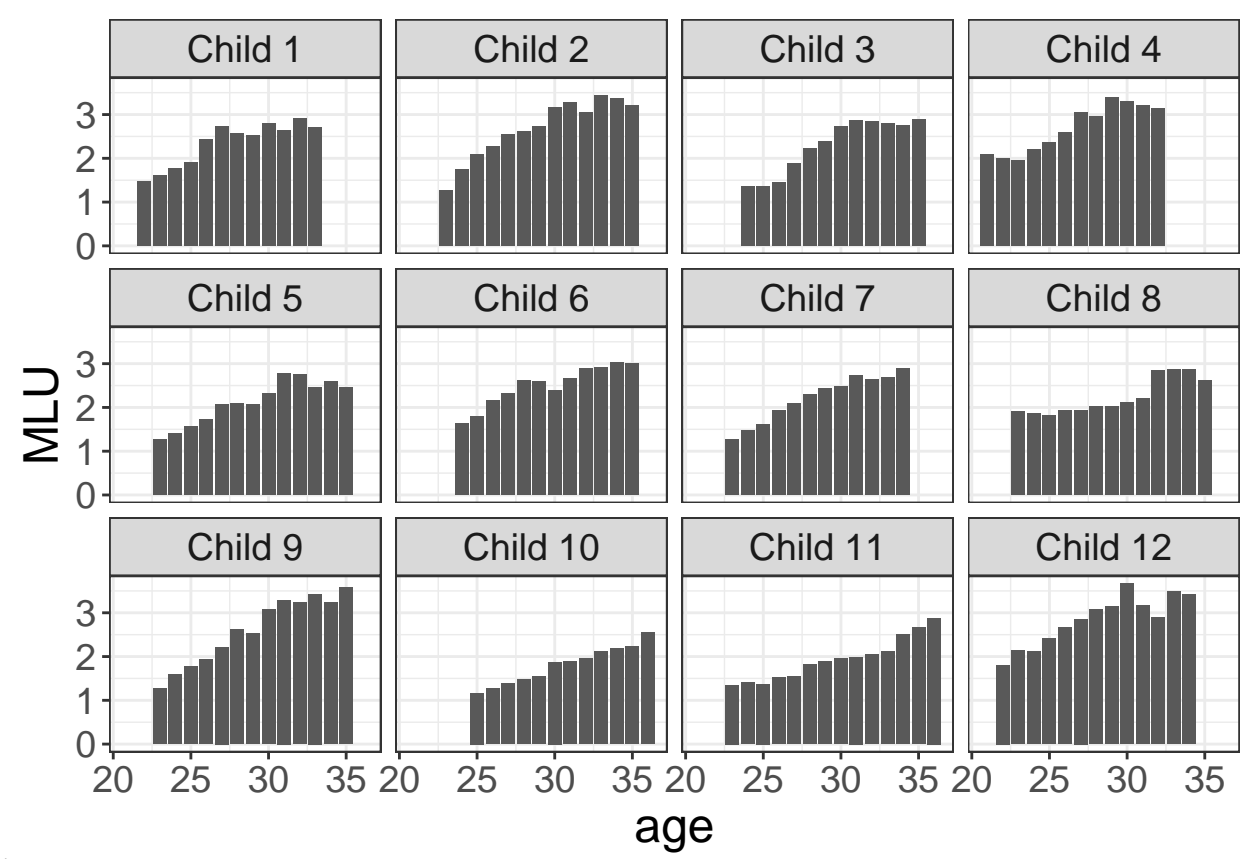

Figure S1

Development of $M L U$ in child speech

relatively stable in CDS, MLU in CS shows an uptrend for and eventually reaching around 3 for most children.

\subsection{Vocabulary size}

Fig. S3 and Fig. S4 show the vocabulary size in merged data sets that were used in the training of word embeddings models. Since we set the minimum count to 2 in the models, the vocabulary only includes words that occur at least twice in the data set. This is essentially the number of words for which the model generates vectors. It is shown in the figures that the vocabulary sizes, after the operation of the sliding window, do not monotonically grow over time. Even in child speech, vocabulary at an older age can be slightly smaller. In addition, vocabulary sizes do not exhibit the similar stages as we have discovered in segmented analysis. 

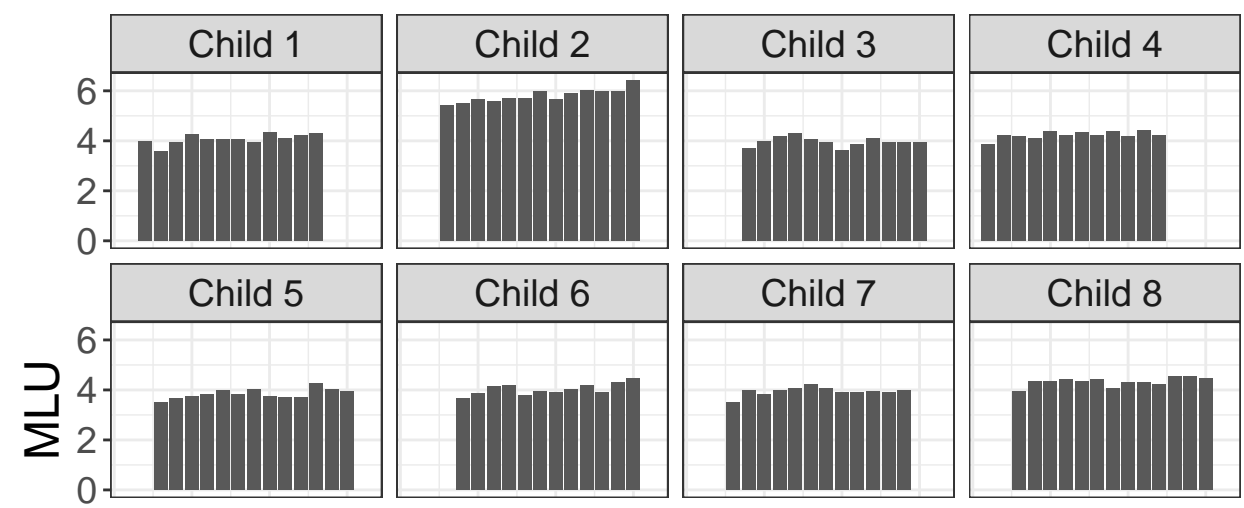

Child 8
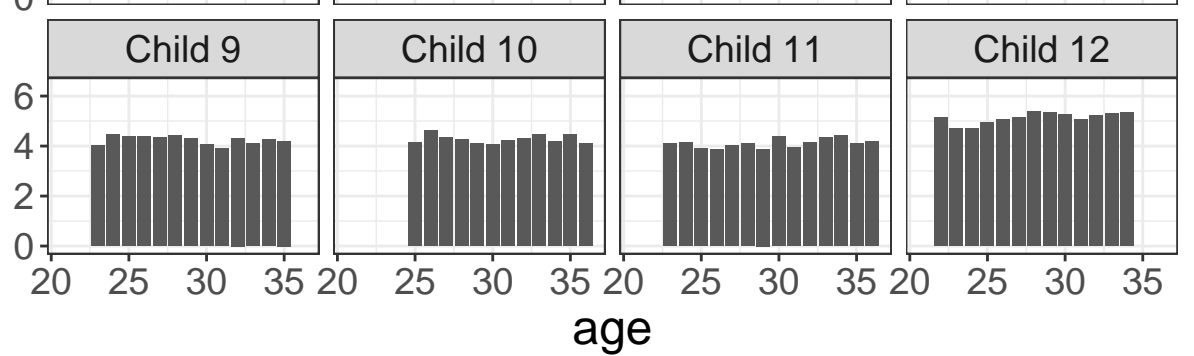

Figure S2

Development of $M L U$ in child-directed speech
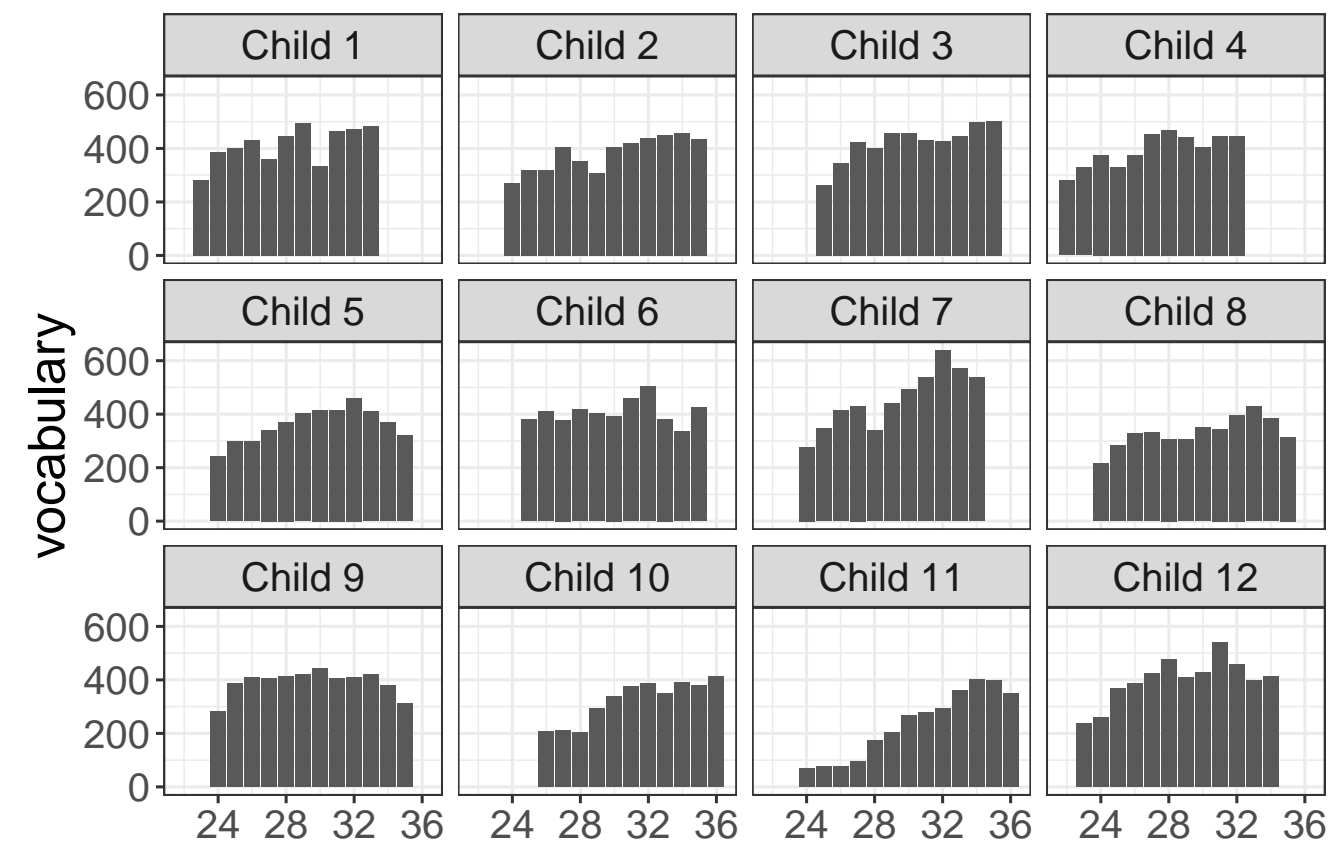

Figure S3

age

Development of vocabulary size (minimum count 2) in child speech 


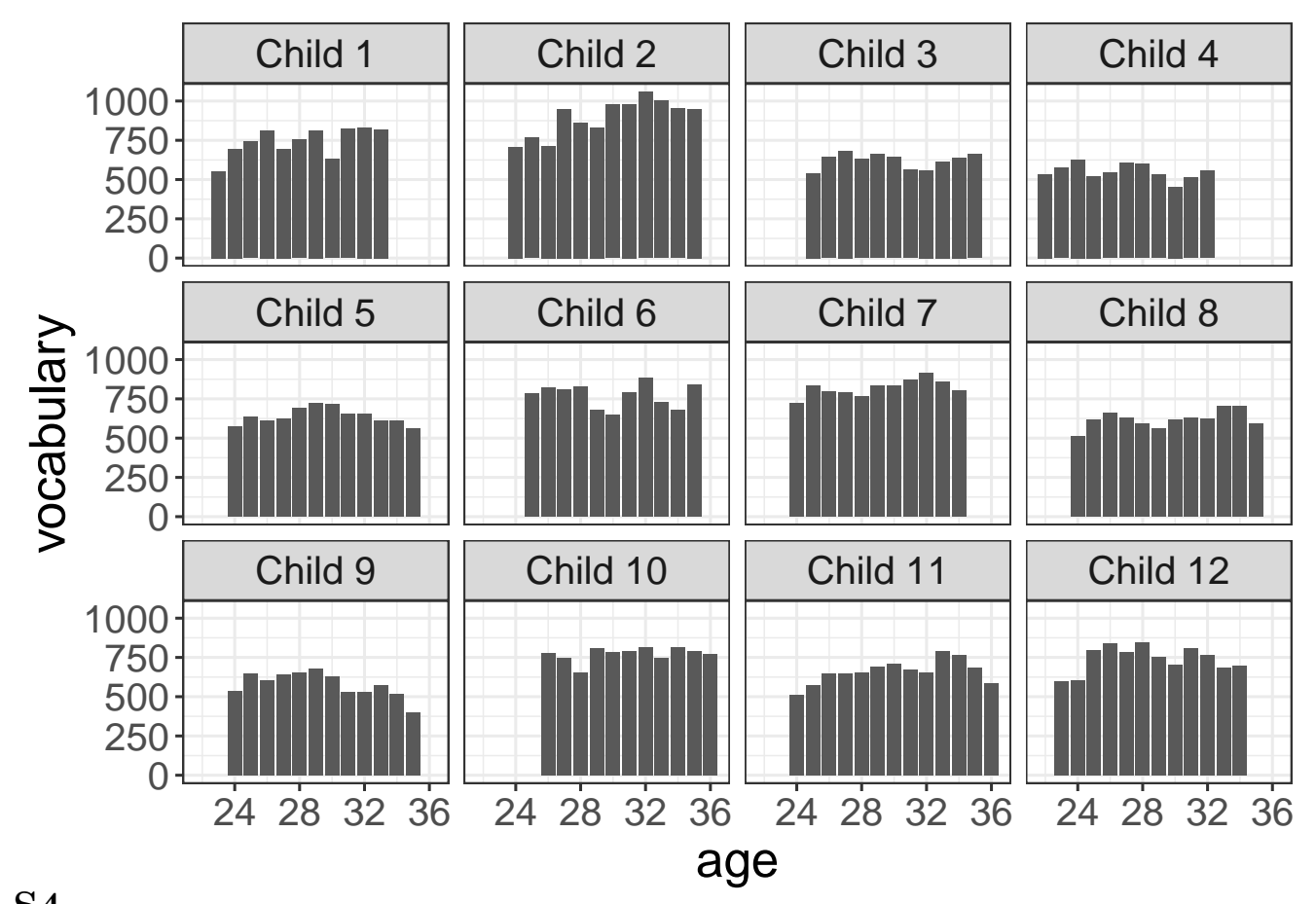

Figure S4

Development of vocabulary size (minimum count 2) in child-directed speech 
2. Models

2.1 Posterior predictive checking for best models

\section{Figure S5}

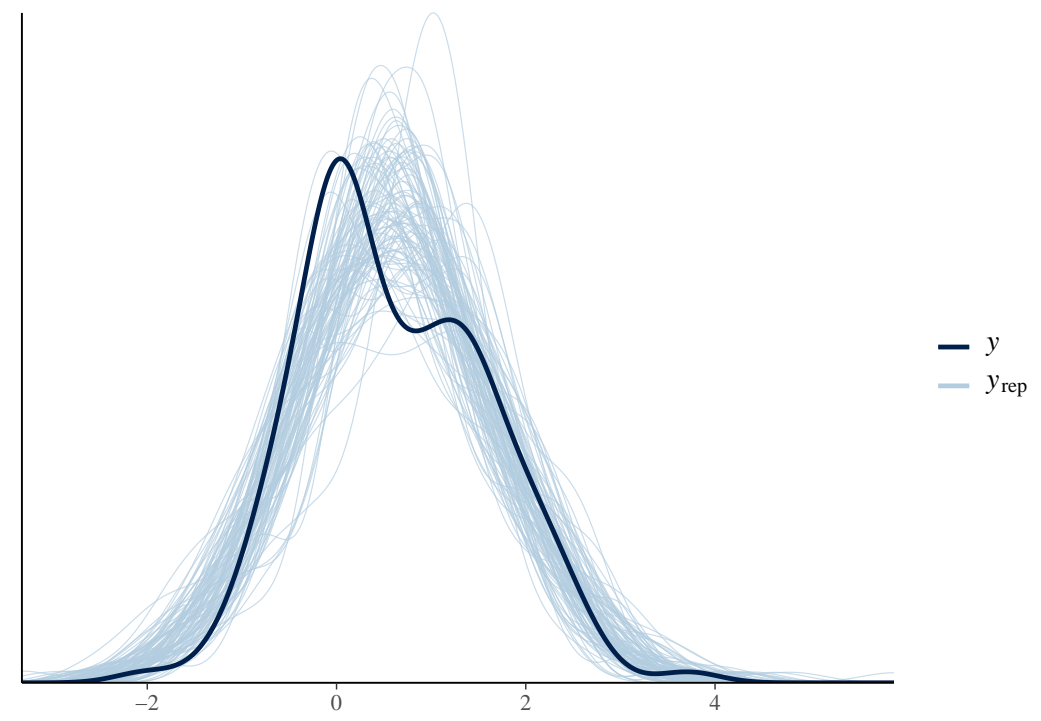

Posterior predictive checking for the best model in child speech

\section{Figure S6}

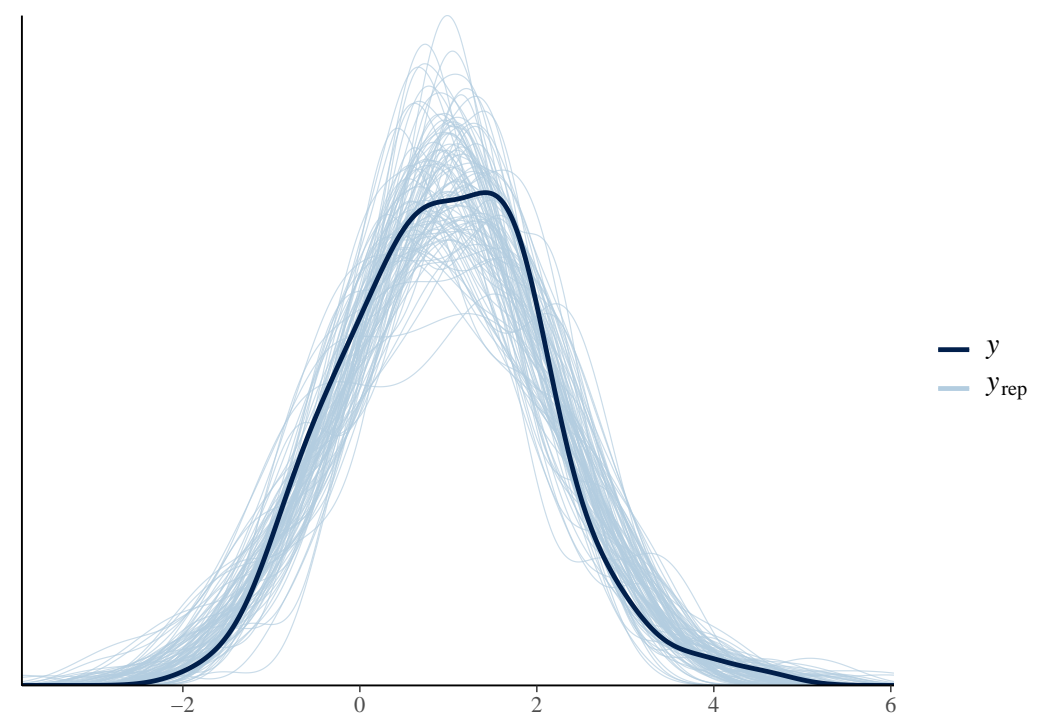

Posterior predictive checking for the best model in child-directed speech 


\section{Figure S7}

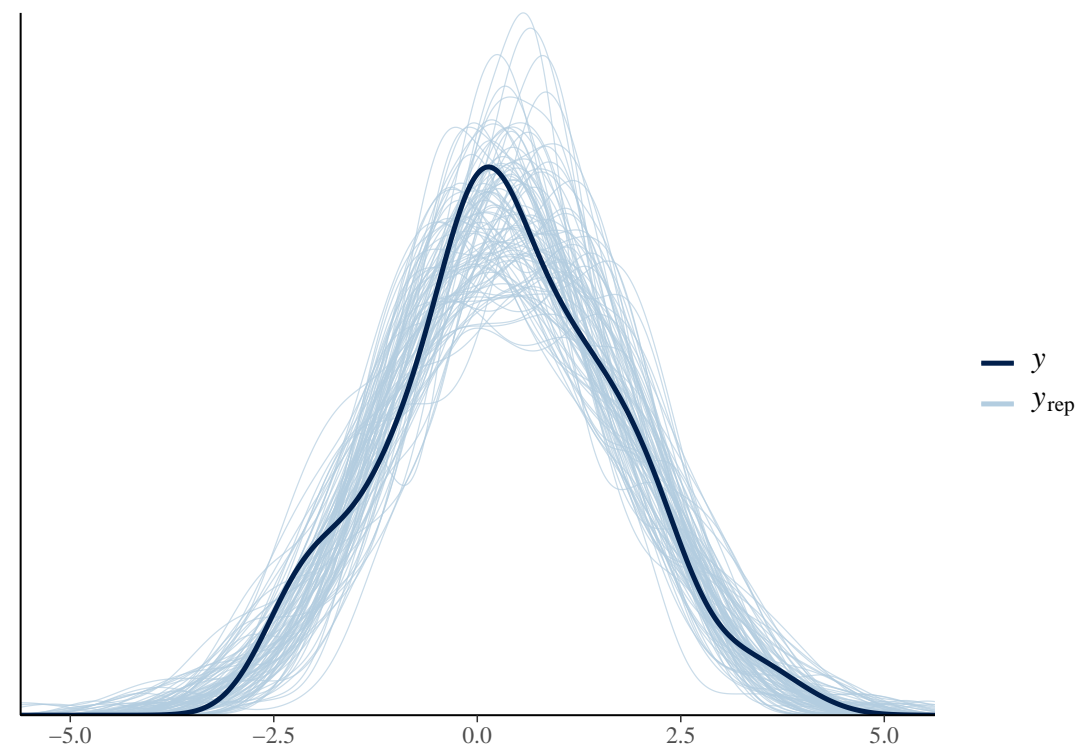

Posterior predictive checking for the model of complexity difference without age as a predictor

\section{Figure S8}

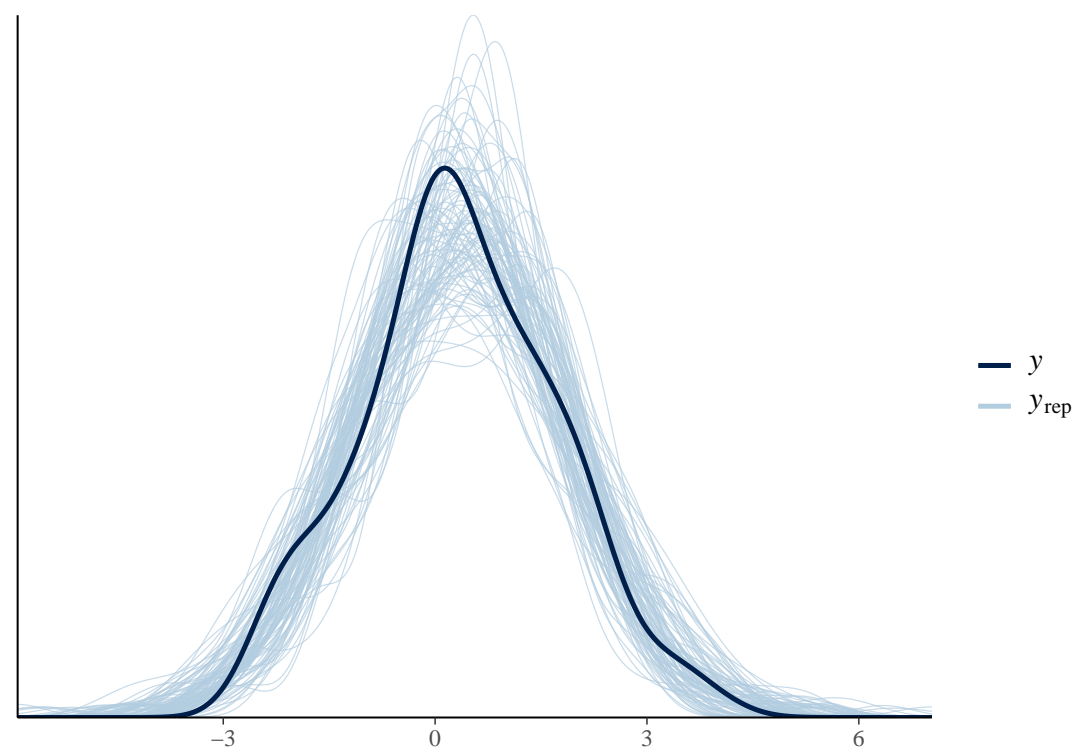

Posterior predictive checking for the model of complexity difference with break points at 27 and 30 months of age 


\subsection{Examination of individual effects}

We examine the age effect at an individual level by including the random slopes. 90\% quantile-based intervals are reported. For child speech, both the decline of causative complexity in the second stage and the resumption growth in the third stage are particularly prominent, with all individuals displaying negative effect and positive effect by age respectively. In general, individuals do not substantially differ between each other in terms of the developmental trends in each stage.

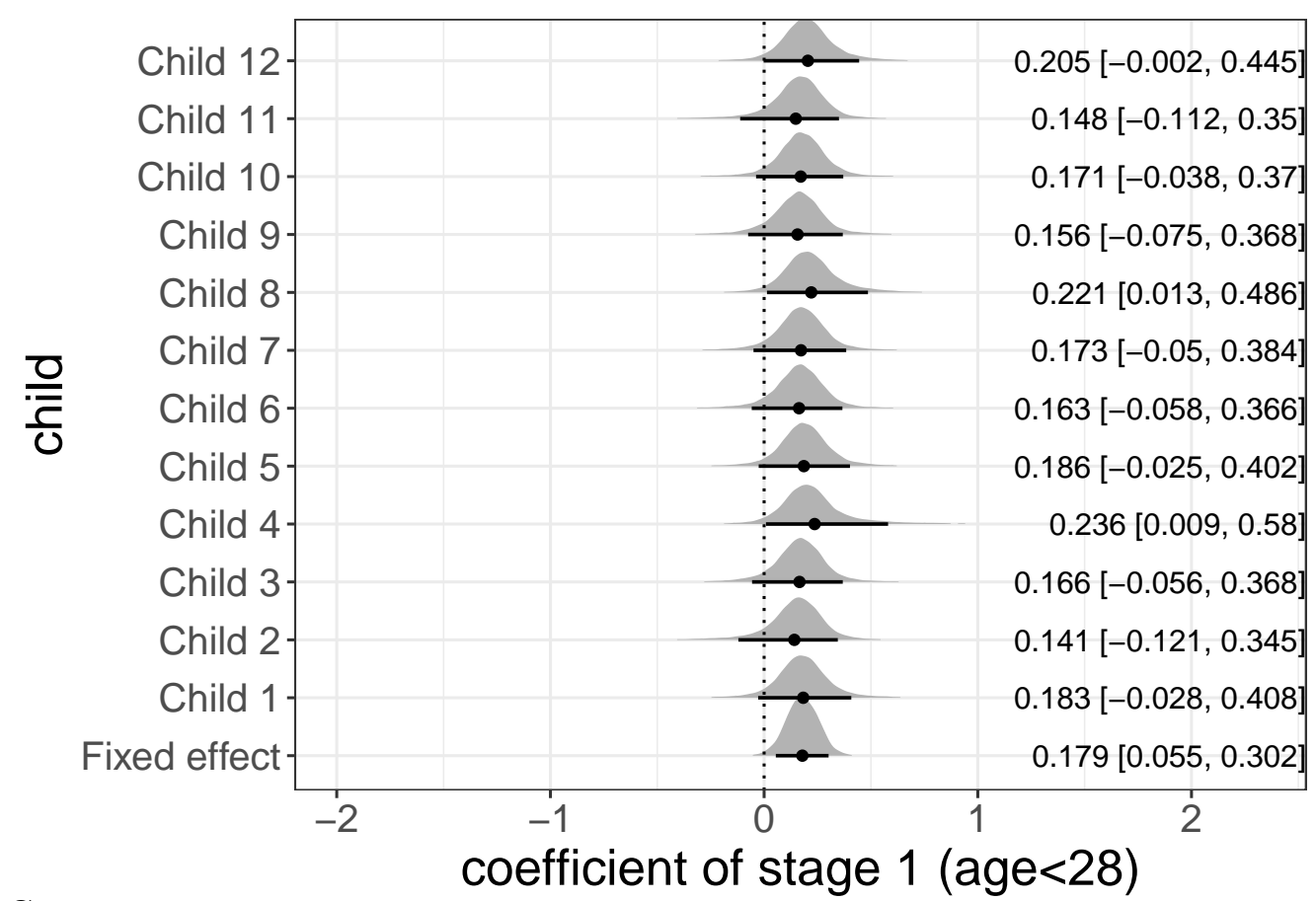

\section{Figure S9}

Age effect in child speech at individual levels by incorporating the random slopes: stage 1 


\section{Figure S10}

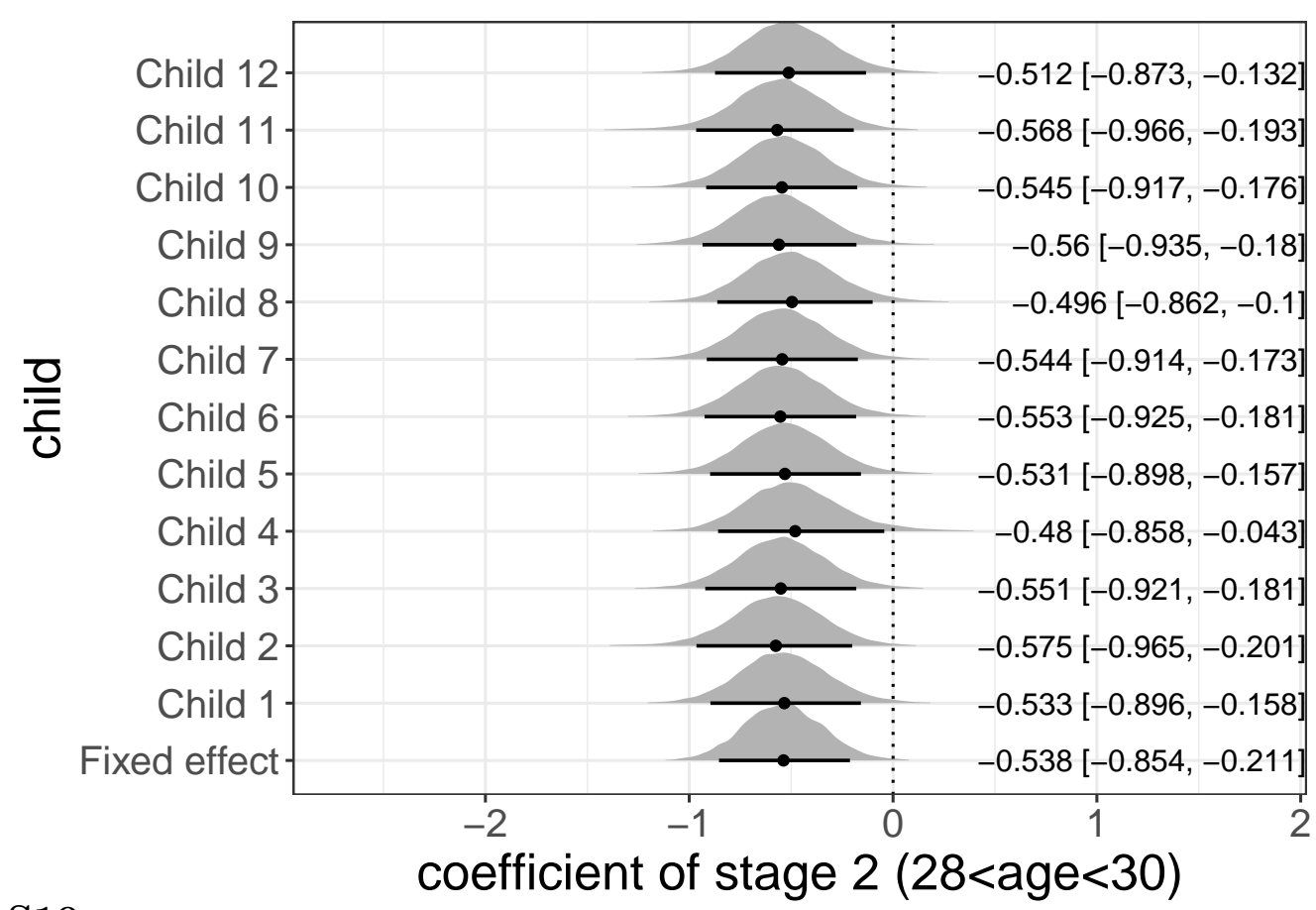

Age effect in child speech at individual levels by incorporating the random slopes: stage 2

\section{Figure S11}

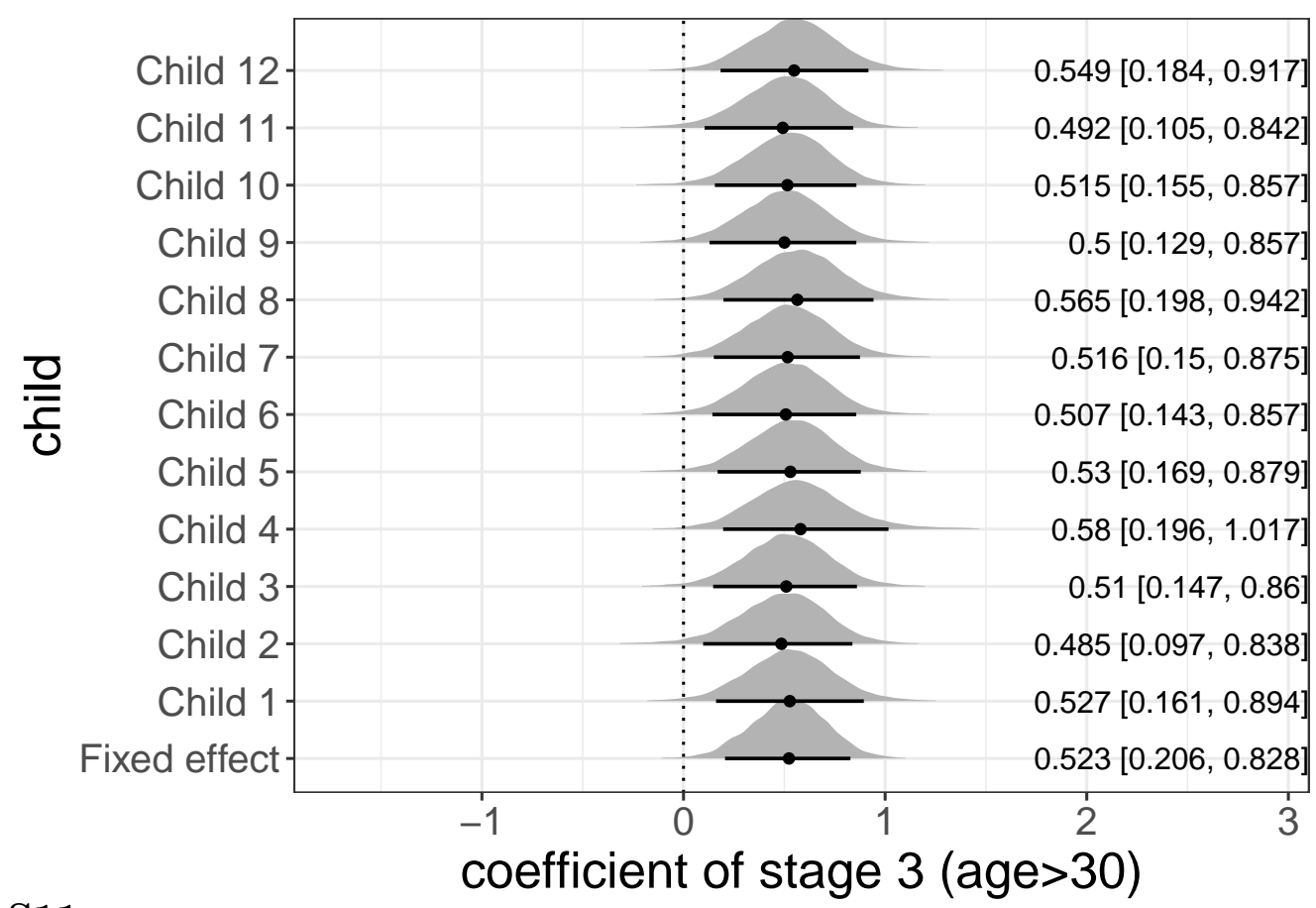

Age effect in child speech at individual levels by incorporating the random slopes: stage 3 
For child-directed speech, variability between individuals is greater than in child speech. The trends are, nonetheless, similar across different children, especially in the second and third stages, with a wider credible interval for some individuals.

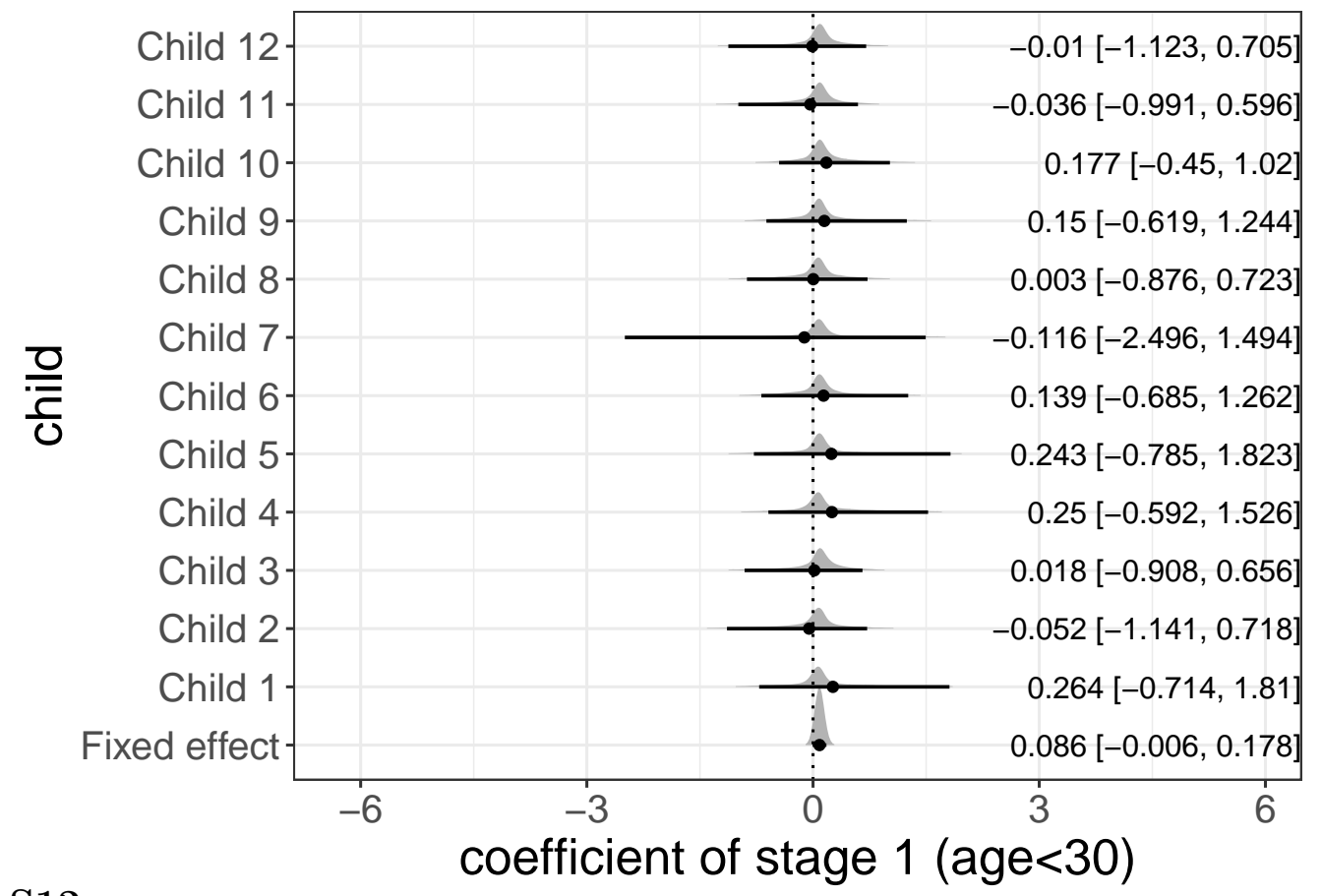

\section{Figure S12}

Age effect in child-directed speech at individual levels by incorporating the random slopes: stage 1 


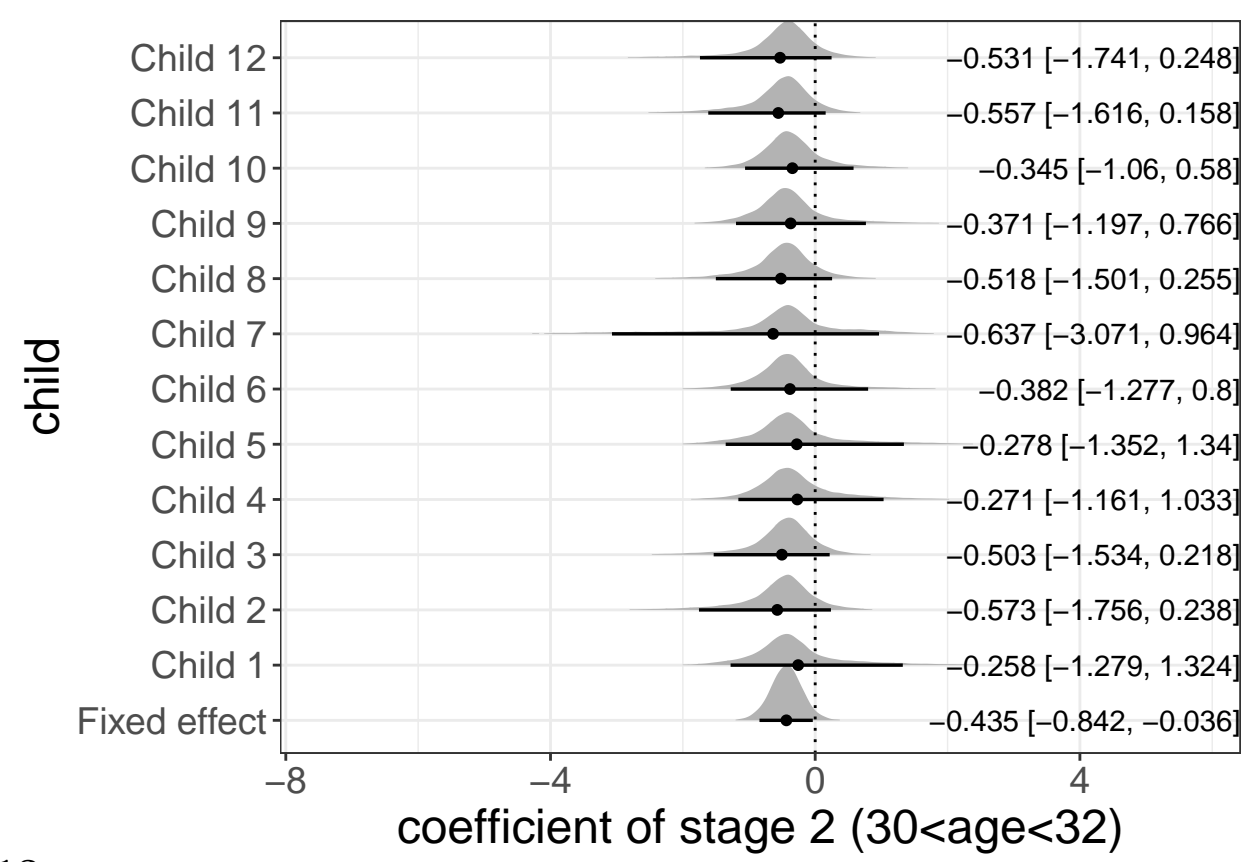

\section{Figure S13}

Age effect in child-directed speech at individual levels by incorporating the random slopes: stage 2

\section{Figure S14}

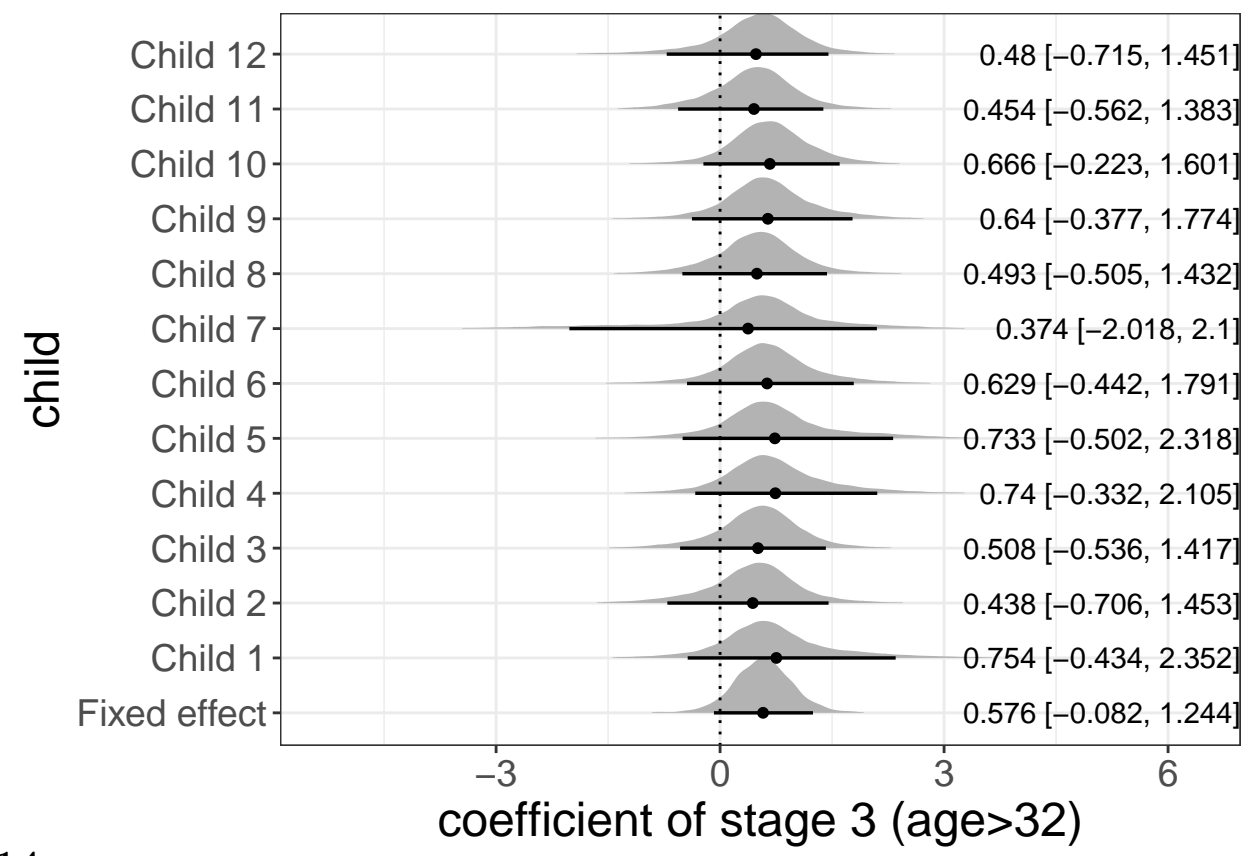

Age effect in child-directed speech at individual levels by incorporating the random slopes: stage 3 
We also examine the model with break points at 27 and 30 months of age in Analysis 2. The increase of the gap between CDS and CS is clear in the second stage for all children, as is the decrease of the gap starting from the age of 30 months, when the adaptation in CDS begins to show.

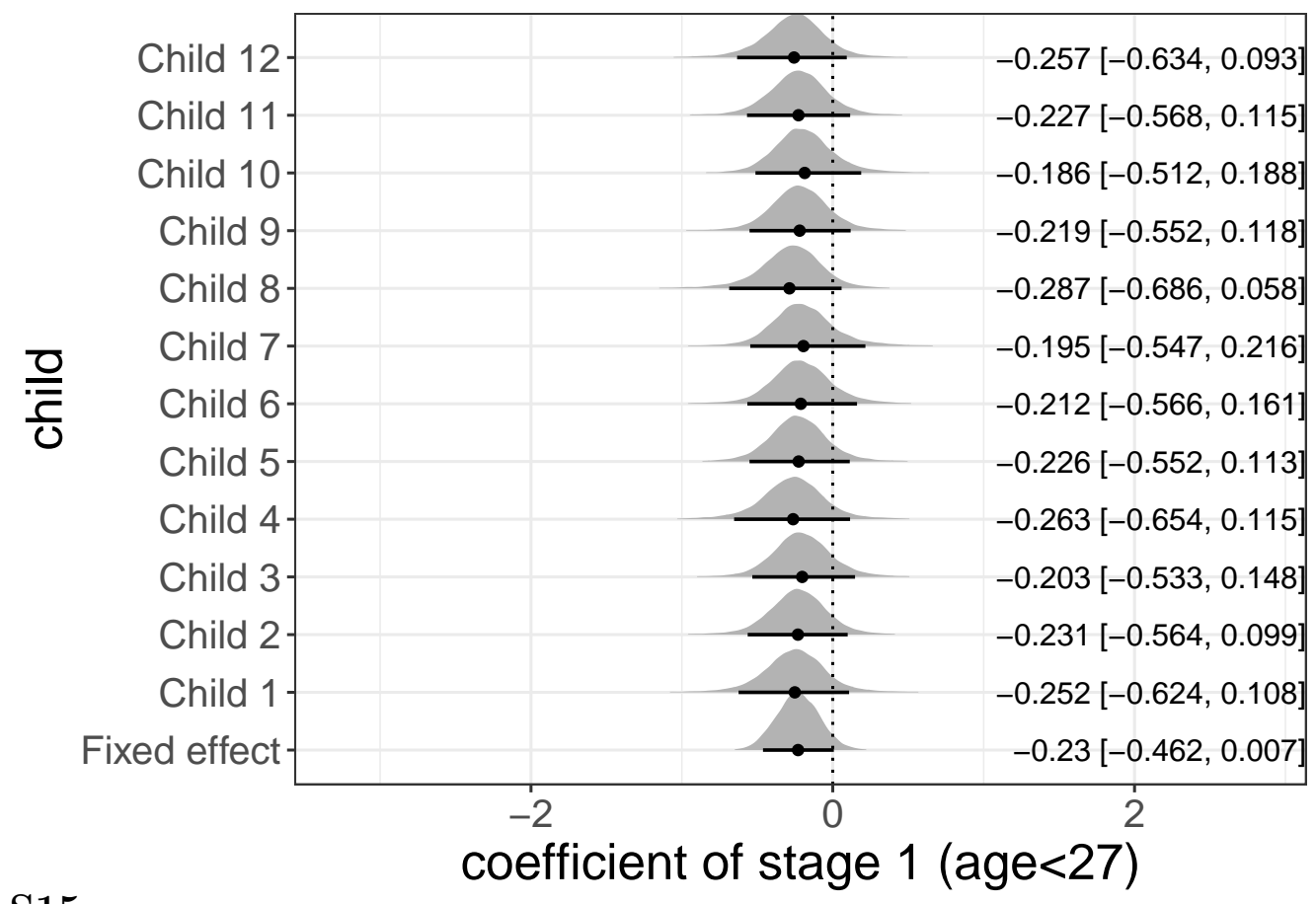

Figure S15

Age effect for complexity difference at individual levels by incorporating the random slopes: stage 1 


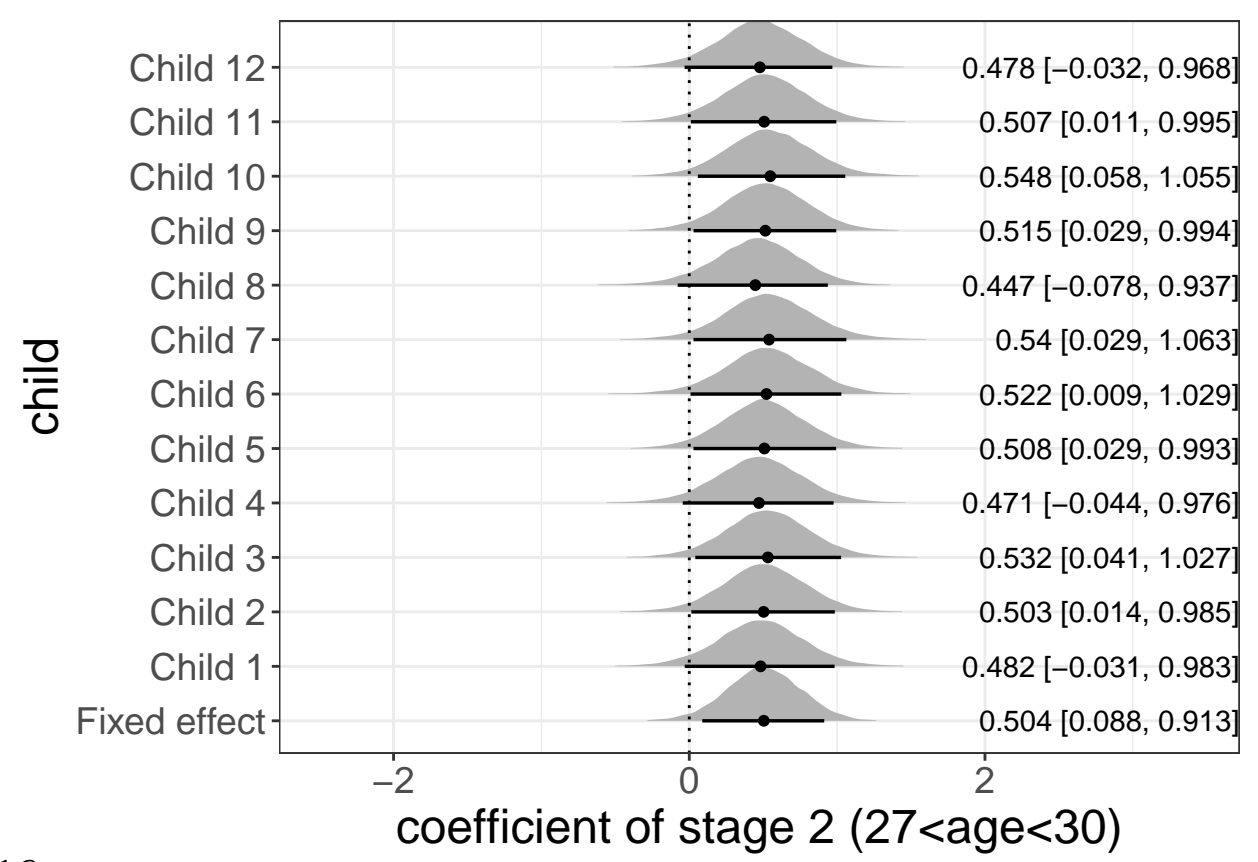

\section{Figure S16}

Age effect for complexity difference at individual levels by incorporating the random slopes: stage 2

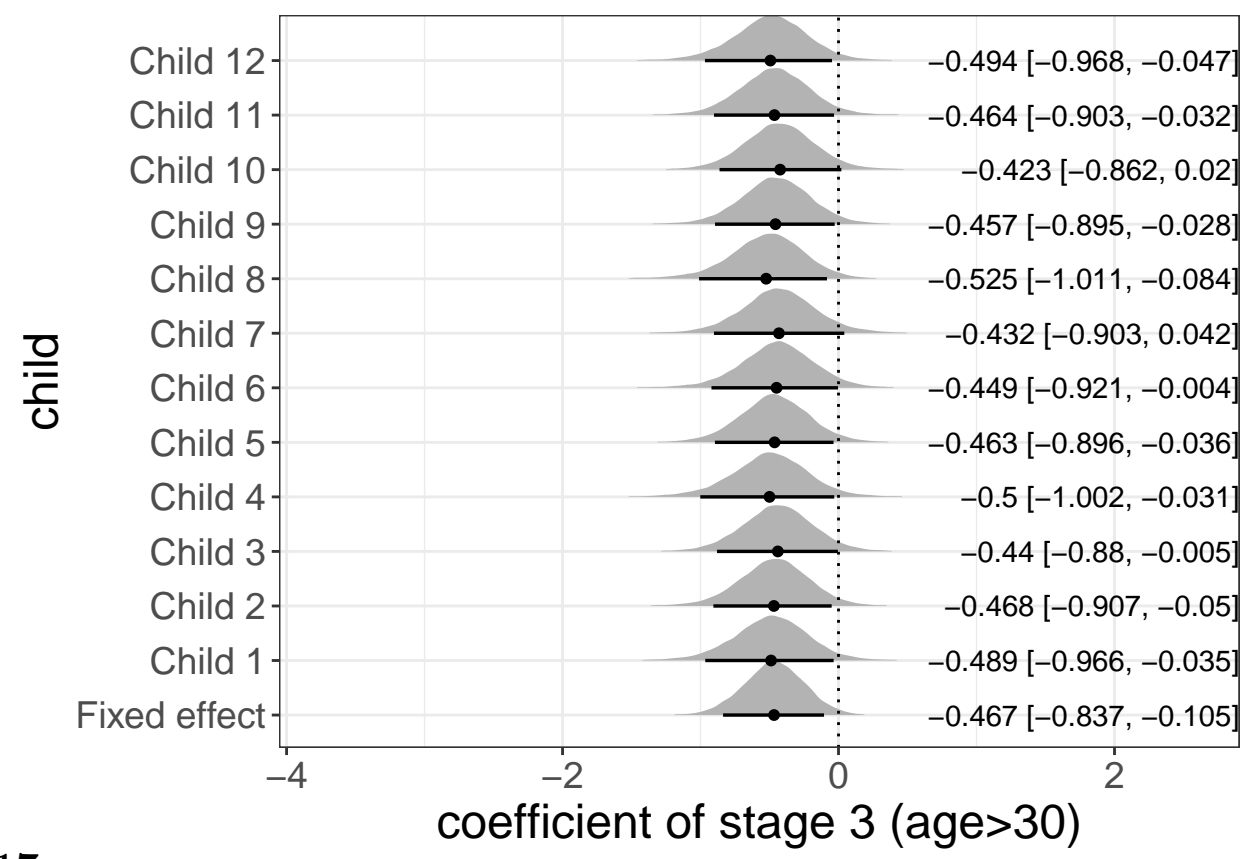

\section{Figure S17}

Age effect for complexity difference at individual levels by incorporating the random slopes: stage 3 